\title{
Relations Between Spatial Calculi About Directions and Orientations
}

Till Mossakowski

Otto-von-Guericke-University of Magdeburg, Faculty of Computer Science

Universitätsplatz 2

39106 Magdeburg

\section{Reinhard Moratz}

University of Maine, National Center for Geographic Information and Analysis, School of Computing and Information Science, 348 Boardman Hall, Orono, 04469 Maine, USA.
TILL@IWS.CS.UNI-MAGDEBURG.DE

REINHARD.MORATZ@MAINE.EDU

\begin{abstract}
Qualitative spatial descriptions characterize essential properties of spatial objects or configurations by relying on relative comparisons rather than measuring. Typically, in qualitative approaches only relatively coarse distinctions between configurations are made. Qualitative spatial knowledge can be used to represent incomplete and underdetermined knowledge in a systematic way. This is especially useful if the task is to describe features of classes of configurations rather than individual configurations.

Although reasoning with them is generally NP-hard (even $\exists \mathbb{R}$-complete), relative directions are important because they play a key role in human spatial descriptions and there are several approaches how to represent them using qualitative methods. In these approaches directions between spatial locations can be expressed as constraints over infinite domains, e.g. the Euclidean plane. The theory of relation algebras has been successfully applied to this field. Viewing relation algebras as universal algebras and applying and modifying standard tools from universal algebra in this work, we (re)define notions of qualitative constraint calculus, of homomorphism between calculi, and of quotient of calculi.Based on this method we derive important properties for spatial calculi from corresponding properties of related calculi. From a conceptual point of view these formal mappings between calculi are a means to translate between different granularities.
\end{abstract}

\section{Introduction}

A qualitative representation of space and/or time provides mechanisms which characterize the essential properties of objects or configurations. The advantages over quantitative representations can be: (1) a better match with human concepts related to natural language, and (2) better efficiency for reasoning. The two main trends in qualitative spatial constraint reasoning (Ligozat, 2011) are topological reasoning about regions (Randell \& Cohn, 1989; Randell, Cui, \& Cohn, 1992; Egenhofer \& Franzosa, 1991; Renz \& Nebel, 1999; Worboys \& Clementini, 2001) and reasoning about directions between points and straight lines and orientations of straight lines or configurations derived from points (Frank, 1991; Ligozat, 1998; Renz \& Mitra, 2004; Freksa, 1992; Clementini, Felice, \& Hernandez, 1997; Scivos \& 
Nebel, 2004; Moratz, Lücke, \& Mossakowski, 2011; Mossakowski \& Moratz, 2012; Dubba, Bhatt, Dylla, Cohn, \& Hogg, 2015).

In constraint-based reasoning about spatial configurations, typically a partial initial knowledge of a scene is represented in terms of qualitative constraints between spatial objects. Implicit knowledge about spatial relations is then derived by constraint propagation. Previous research has found that the mathematical notion of a relation algebra and related notions are well-suited for this kind of reasoning. In particular, in an arbitrary relation algebra, the well-known path consistency algorithm (Montanari, 1974) computes an algebraic closure of a given constraint network, and this approximates, and in many cases also decides, consistency of the network in polynomial time. Intelligent backtracking techniques and the study of maximal tractable subclasses also allow for efficiently deciding networks involving disjunctions. Starting with Allen's temporal interval algebra, this approach has been successfully applied to several qualitative constraint calculi, and is now supported by freely available toolboxes (Gantner, Westphal, \& Wölfl, 2008; Wallgrün, Frommberger, Wolter, Dylla, \& Freksa, 2006). Moreover, people have started to develop benchmark problem libraries (Nebel \& Wölll, 2009) and have shown that this method performs quite well also when compared to other constraint reasoning techniques (Westphal \& Wölfl, 2009).

In this work, we apply universal algebraic tools to qualitative calculi. This connection has been previous investigated in the literature (Li, Kowalski, Renz, \& Li, 2008; Bodirsky, 2008; Huang, 2012). However, in our paper we deviate from standard universal algebra by using lax and oplax homomorphisms, which have weaker properties than standard homomorphisms (and more an order-theoretic than algebraic flavor), but are better suited for transferof algebraic structure between qualitative calculi such as $\mathcal{D} \mathcal{R} \mathcal{A}_{f p}, \mathcal{O P} \mathcal{R} \mathcal{A}_{1}^{*}$ and $\mathcal{C Y \mathcal { C }}{ }_{b}$. In this work, we focus on calculi of binary relations only.

\section{Relation Algebras for Spatial Reasoning}

Standard methods developed for finite domains generally do not apply to constraint reasoning over infinite domains. The theory of relation algebras (Ladkin \& Maddux, 1994; Maddux, 2006) allows for a purely symbolic treatment of constraint satisfaction problems involving relations over infinite domains. The corresponding constraint reasoning techniques were originally introduced by Montanari (1974), applied for temporal reasoning (Allen, 1983) and later proved to be valuable for spatial reasoning (Renz \& Nebel, 1999; Isli \& Cohn, 2000). The central data for a binary calculus is given by:

- a list of (symbolic names for) base-relations, which are interpreted as relations over some domain, having the crucial "JEPD" properties of joint exhaustiveness and pairwise disjointness (a general relation is then simply a union of base-relations).

- a table for the computation of the converses of relations.

- a table for the computation of the compositions of relations.

Then, the path consistency algorithm (Montanari, 1974) and backtracking techniques (van Beek \& Manchak, 1996) are the tools used to tackle the problem of consistency of constraint networks and related problems. These algorithms have been implemented in both generic reasoning toolboxes GQR (Gantner, Westphal, \& Wölfl, 2008) and SparQ (Wallgrün et al., 
2006). To integrate a new calculus into these tools, only a list of base-relations and tables for compositions and converses (plus a compositional identity, which however is not really used) need to be provided. Thereby, the qualitative reasoning facilities of these tools become available for this calculus. ${ }^{1}$ Since the compositions and converses of general relations can be reduced to compositions and converses of base-relations, these tables only need to be given for base-relations. Based on these tables, the tools provide a means to approximate the consistency of constraint networks, list all their atomic refinements, and more (see Section 4 for some details).

Let $b$ be a base-relation. The converse $b^{\smile}=\{(x, y) \mid(y, x) \in b\}$ is often itself a baserelation. Since base-relations generally are not closed under composition, this operation is approximated by a weak composition:

$$
b_{1} \diamond b_{2}=\bigcup\left\{b \text { base-relation } \mid\left(b_{1} \circ b_{2}\right) \cap b \neq \emptyset\right\}
$$

where $b_{1} \circ b_{2}$ is the usual set theoretic composition

$$
b_{1} \circ b_{2}=\left\{(x, z) \mid \exists y \cdot(x, y) \in b_{1},(y, z) \in b_{2}\right\}
$$

Composition is said to be strong if $b_{1} \diamond b_{2}=b_{1} \circ b_{2}$ for all base-relations $b_{1}, b_{2}$. Generally, $b_{1} \diamond b_{2}$ over-approximates the set-theoretic composition, while a strong composition captures it exactly.

The mathematical background of composition in table-based reasoning is given by the theory of relation algebras (Maddux, 2006; Renz \& Nebel, 2007). For many calculi, including the dipole calculus (see Ex. 9 below), a slightly weaker notion is needed, namely that of a non-associative algebra (Maddux, 2006; Ligozat \& Renz, 2004), where associativity has been dropped. These algebras treat spatial relations as abstract entities (independently of any domain) that can be combined by certain operations and governed by certain equations.

Definition 1 (Maddux 2006; Ligozat \& Renz 2004). A non-associative algebra $A$ is a tuple $A=(A, \vee,-, \wedge, 0,1, \diamond, \smile, \Delta)$ such that:

1. $(A, \vee,-, \wedge, 0,1)$ is a Boolean algebra. $\vee$ is called join, $\wedge$ meet, 0 bottom, 1 top, and - relative complement. Note each Boolean algebra carries a partial order defined by $a \leq b$ iff $a \vee b=b$;

2. $\Delta$ is a constant (called identity relation), ` a unary operation (called converse) and $\diamond$ a binary operation (called weak composition) such that, for any $a, b, c \in A$ :
(a) $\left(a^{\smile}\right)^{\smile}=a$
(b) $\Delta \diamond a=a \diamond \Delta=a$
$(c) a \diamond(b \vee c)=a \diamond b \vee a \diamond c$
(d) $(a \vee b)^{\smile}=a^{\smile} \vee b^{\smile}$
(e) $(a-b)^{\smile}=a^{\smile}-b^{\smile}$
(g) $(a \diamond b) \wedge c^{\smile}=0$ if and only if $(b \diamond c) \wedge a^{\smile}=0$
$(f)(a \diamond b)^{\smile}=b^{\smile} \diamond a^{\smile}$

A non-associative algebra is called a relation algebra, if weak composition $\diamond$ is associative. ${ }^{2}$

1. With more information about a calculus, both of the tools can provide functionality that goes beyond simple qualitative reasoning for constraint calculi.

2. This terminology is a bit misleading, since relation algebras are associative "non-associative algebras". A more precise name for non-associative algebras would be "relation algebras without associativity requirement". Nevertheless, we stick to the terminology established in the literature. 
The elements of such an algebra will be called (abstract) relations. We are mainly interested in finite non-associative algebras that are complete and atomic, which means that there is a set of pairwise disjoint minimal relations, the atoms, also called base-relations, and all relations can be obtained as joins of these. Then, the following fact is well-known and easy to prove:

Proposition 2 (Düntsch, 2005). A complete atomic non-associative algebra is uniquely determined by its set of base-relations, together with the converses and compositions of baserelations. (Note that the composition of two base-relations is in general not a base-relation.)

When providing examples, it is easier to start with partition schemes:

Definition 3 (Ligozat \& Renz, 2004; Mossakowski et al., 2006). Let $U$ be a non-empty set. A partition scheme on $U$ is defined by a finite (index) set $I$ with a distinguished element $i_{0} \in I$, a unary operation ${ }^{`}$ on $I$, and a family of binary relations $\left(R_{i}\right)_{i \in I}$ on $U$ such that

1. $\left(R_{i}\right)_{i \in I}$ is a partition of $U \times U$ in the sense that the $R_{i}$ are pairwise disjoint and jointly exhaustive.

2. $R_{i_{0}}$ is the diagonal relation $\{(x, x) \mid x \in U\}$.

3. $R_{i}$ is the (set-theoretical) converse of relation $R_{i}$, for each $i \in I$.

The relations $R_{i}$ are referred to as basic relations. In the following we often write

$$
U \times U=\bigcup_{i \in I} R_{i}
$$

to denote partition schemes.

Proposition 4 (Ligozat \& Renz, 2004; Mossakowski et al., 2006). Given a partition scheme

$$
U \times U=\bigcup_{i \in I} R_{i}
$$

we obtain a non-associative algebra as follows: the Boolean algebra component is $\mathcal{P}(I)$, the powerset of $I$. The converse is given by pointwise application of ${ }^{\smile}$; the diagonal is $i_{0}$. Composition is given by weak composition as defined above.

We now introduce several qualitative calculi by just giving its domain $U$ and its set of basic relations; the diagonal and the converse are clear.

Example 5. The most prominent temporal calculus is Allen's interval algebra $\mathcal{I} \mathcal{A}$ (Allen, 1983), which describes possible relations between intervals in linear flows of time ${ }^{3}$. An interval is a pair $(s, t)$ of real numbers such that $s<t$. The 13 basic relations between such intervals are depicted in Fig. 1.

3. There is also a spatial interpretation of the Allen calculus in which the intervals are interpreted as one-dimensional spatial entities 


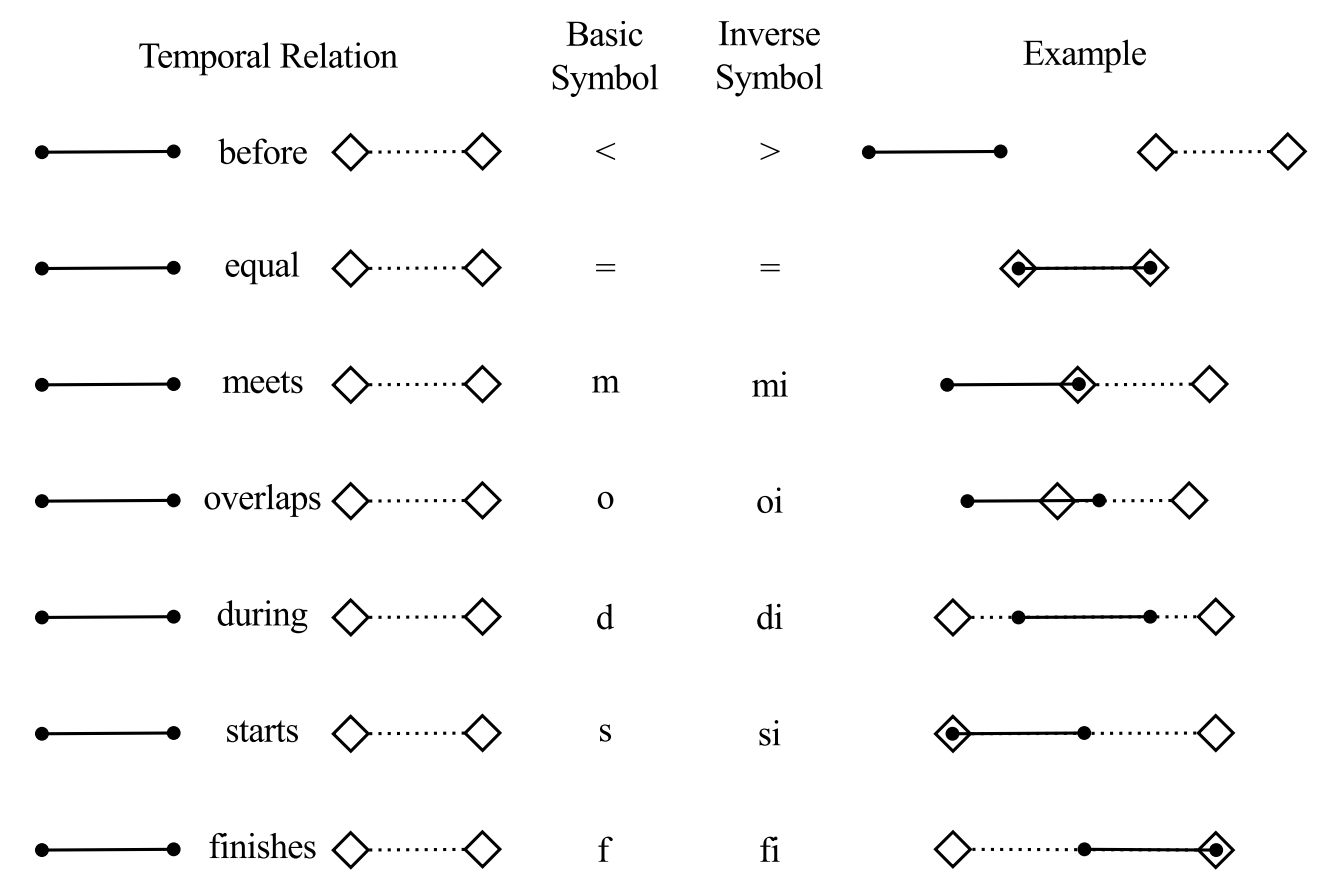

Figure 1: Allen's interval relations

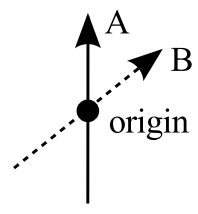

ArB

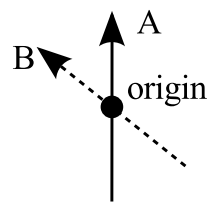

A1B

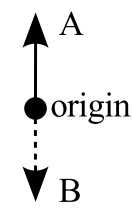

Ao B

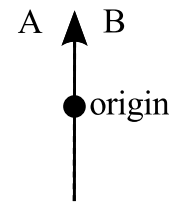

A e B

Figure 2: $\mathcal{C Y C}_{b}$ relations. "A r B" means "B is to the right of $\mathrm{A}$ ".

Example 6. The $\mathcal{C} \mathcal{Y} \mathcal{C}_{b}$ calculus (Isli \& Cohn, 2000) is based on the domain $\mathbb{C Y} \mathbb{C}=\{\phi \mid$ $-\pi<\phi \leq \pi\}$ of cyclic orientations. Equivalently, these angles can be represented as oriented straight lines containing the origin of the 2D Euclidian plane associated with a reference system. Using this latter representation, Fig. 2 depicts the four base-relations r, l, o, e (e.g. "right", "left", "opposite", "equal") of $\mathcal{C} \mathcal{Y} \mathcal{C}_{b}$.

The converse and composition tables are as follows:

\begin{tabular}{|l||l|}
\hline$b$ & $b^{\smile}$ \\
\hline \hline$e$ & $e$ \\
\hline$l$ & $r$ \\
\hline$o$ & $o$ \\
\hline$r$ & $l$ \\
\hline
\end{tabular}

\begin{tabular}{|c||c|c|c|c|}
\hline$\circ$ & $e$ & $l$ & $o$ & $r$ \\
\hline \hline$e$ & $e$ & $l$ & $o$ & $r$ \\
\hline$l$ & $l$ & $\{l, o, r\}$ & $r$ & $\{e, l, r\}$ \\
\hline$o$ & $o$ & $r$ & $e$ & $l$ \\
\hline$r$ & $r$ & $\{e, l, r\}$ & $l$ & $\{l, o, r\}$ \\
\hline
\end{tabular}


Example 7. The $\mathcal{O P} \mathcal{R} \mathcal{A}_{n}$ calculus (Moratz, 2006; Mossakowski \& Moratz, 2012) is based on the domain $\mathbb{O P}=\left\{(p, \phi) \mid p \in \mathbb{R}^{2},-\pi<\phi \leq \pi\right\}$ of oriented points in Euclidean plane. An oriented point consists of a point and an angle serving as its orientation. The full angle is divided using $n$ axes, leading to $4 n$ regions, see Fig. 3 . If the points of $A$ and $B$ differ, the

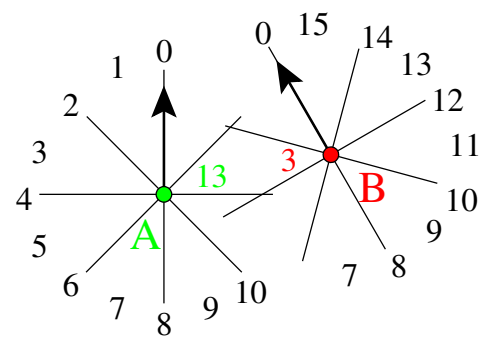

Figure 3: Two o-points in relation $A_{4} \angle_{13}^{3} B$

relation $A_{m} \angle_{i}^{j} B\left(i, j \in \mathcal{Z}_{4 m}{ }^{4}\right)$ reads like this: given a granularity $m$, the relative position of $B$ with respect to $A$ is described by $i$ and the relative position of $A$ with respect to $B$ is described by $j$. If the points of $A$ and $B$ coincide, the relation $A{ }_{m} \angle i B$ expresses that the difference between $B$ 's and $A$ 's orientations (angles) is in region $i$.

For the special case of the $\mathcal{O P} \mathcal{R} \mathcal{A}_{n}$ calculus with $n=1$ (e.g. $\left.\mathcal{O P} \mathcal{R} \mathcal{A}_{1}\right)$ we have a $\operatorname{cog}$ nitively motivated symbolic notation in addition to the general notation for $\mathcal{O P} \mathcal{R} \mathcal{A}_{n}$ baserelations introduced above. Fig. 4 depicts an oriented point and the corresponding division of the plane into the regions Front, Left, Right and Back and Same (the latter stands for the point itself). The naming schema for the $\mathcal{O P} \mathcal{R} \mathcal{A}_{1}$ base-relations concatenates the name for the relative position of the second oriented point w.r.t. the first and then the relative position of first oriented point w.r.t. the second. Using capitalization of the first part of the relation symbol, the cognitively motivated schema for the relation names leads to these names for the 16 base-relations of $\mathcal{O P} \mathcal{R} \mathcal{A}_{1}$ : FRONTfront, FRONTleft, FRONTright, FRONTback, LEFTfront, LEFTleft, LEFTright, LEFTback, RIGHTfront, RIGHTleft, RIGHTright, RIGHTback, BACKfront, BACKleft, BACKright, and BACKback. Again, if both points coincide, we compare their orientations. This leads to the relations SAMEfront, SAMEleft, SAMEright and SAMEback.

SAMEfront is the identity relation. SAMEback is analogous to the opposite relation of $\mathcal{C Y \mathcal { C }}_{b}$ (see Fig. 2). Also SAMEleft and SAMEright are analogous to the corresponding $\mathcal{C} \mathcal{Y} \mathcal{C}_{b}$ relations.

Example 8. The $\mathcal{O P} \mathcal{R} \mathcal{A}_{m}^{*}$ calculus (Dylla, 2008) is similar to $\mathcal{O P} \mathcal{R} \mathcal{A}_{m}$. Here, we concentrate on $\mathcal{O P} \mathcal{R} \mathcal{A}_{1}^{*}$. The important extension is a refinement that is applied to the relations RIGHTright, RIGHTleft, LEFTleft, and LEFTright. These relations are refined by marking them with letters '+' or '-', 'P' or 'A', according to whether the two orientations of the oriented points are positive, negative, parallel or anti-parallel, similar as in Fig. 6:

- LEFTleft is refined into LEFTleftA, LEFTleft+ and LEFTleft-.

4. $\mathcal{Z}_{4 m}$ is the residue ring; for simplicity, we set $\mathcal{Z}_{4 m}=\{0, \ldots, 4 m-1\}$. 


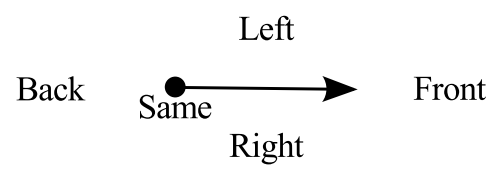

Figure 4: $\mathcal{O} \mathcal{P} \mathcal{R} \mathcal{A}_{1}$ base frame

- LEFT right is refined into LEFTrightP, LEFTright+ and LEFTright-.

- RIGHTright is refined into RIGHTrightA, RIGHTright+ and RIGHTright-.

- RIGHT left is refined into RIGHTleftP, RIGHTleft+ and RIGHTleft-.

The remaining four options LEFTleftP, LEFTrightA, RIGHTrightP and RIGHTleftA are geometrically impossible. Altogether, we obtain a set of 28 base-relations.

Example 9. A dipole is a pair of distinct points in the Euclidean plane. Before explaining dipole-dipole relations, we first study dipole-point relations. We distinguish between whether a point lies to the left, to the right, or at one of five qualitatively different locations on the straight line that passes through the corresponding dipole (Ligozat, 1993; Scivos \& Nebel, 2004). The corresponding regions are shown on the right side of Fig. 5.

Using these seven possible relations between a dipole and a point, the relations between two dipoles may be specified according to the following conjunction of four relationships:

$$
A \mathrm{R}_{1} \mathbf{s}_{B} \wedge A \mathrm{R}_{2} \mathbf{e}_{B} \wedge B \mathrm{R}_{3} \mathbf{s}_{A} \wedge B \mathrm{R}_{4} \mathbf{e}_{A},{ }^{5}
$$

where $\mathrm{R}_{\mathrm{i}} \in\{\mathrm{l}, \mathrm{r}, \mathrm{b}, \mathrm{s}, \mathrm{i}, \mathrm{e}, \mathrm{f}\}$ with $1 \leq i \leq 4$. The formal combination gives us 2401 relations, out of which 72 relations are geometrically possible. These constitute the $\mathcal{D} \mathcal{R} \mathcal{A}_{f}$ calculus (Moratz, Renz, \& Wolter, 2000; Moratz, Lücke, \& Mossakowski, 2011). For example, in Fig. 5, the relation $A \operatorname{lrrr} B$ holds.

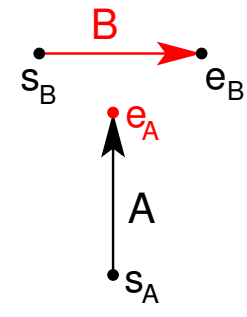

$A \operatorname{Irr} B$

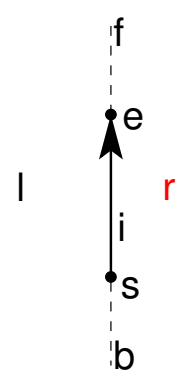

Figure 5: Orientation between two dipoles based on four dipole-point relations

Fig. 6 shows a refinement of $\mathcal{D} \mathcal{R} \mathcal{A}_{f}$, called $\mathcal{D} \mathcal{R} \mathcal{A}_{f p}$, with additional distinguishing features due to parallelism. For the relations different from rrrr, llrr rrll and $11 l 1$, a '+', '-', 'P' or 'A' is already determined by the original base-relation and does not have to be mentioned explicitly. These base-relations then have the same relation symbol as in $\mathcal{D} \mathcal{R} \mathcal{A}_{f}$.

This leads to a set of $80 \mathcal{D} \mathcal{R} \mathcal{A}_{f p}$ base-relations. The relation sese is the identity relation. We denote the resulting non-associative algebra by $\mathcal{D} \mathcal{R} \mathcal{A}_{f p}$.

5. Note that e.g. $A \mathrm{r} \mathrm{s}_{B}$ reads "s $\mathrm{s}_{B}$ is to the right of $A$ ". 


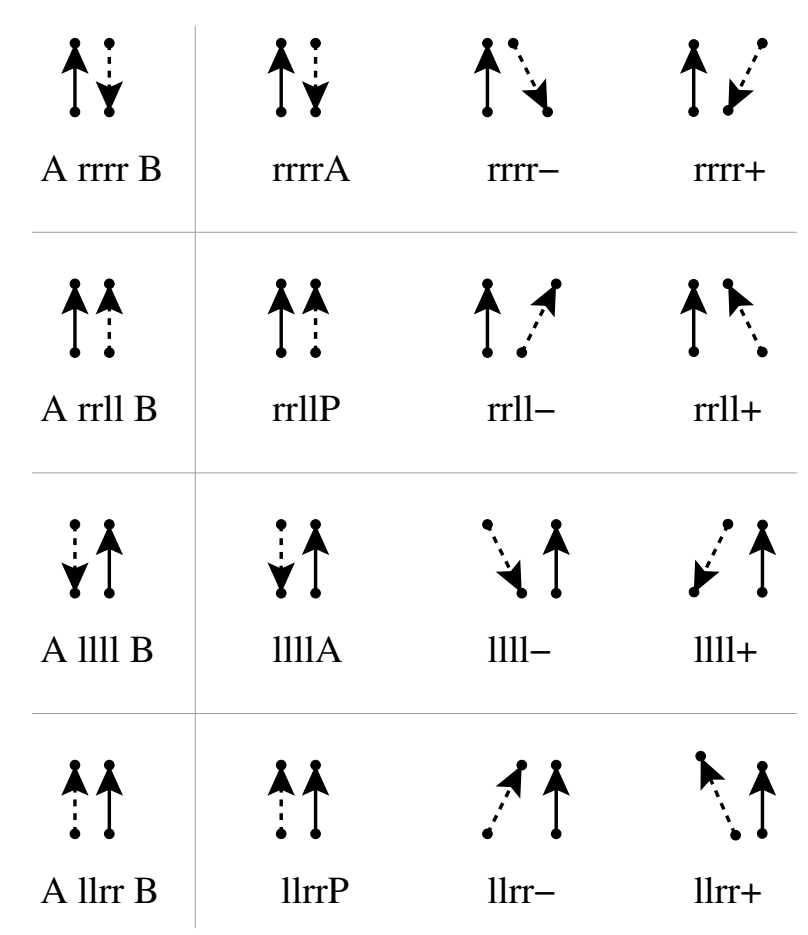

Figure 6: Refined base-relations in $\mathcal{D} \mathcal{R} \mathcal{A}_{f p}$. The solid arrow denotes $A$, the dashed arrow denotes $B$.

\section{Homomorphisms and Weak Representations}

The presented calculi offer the possibility to describe scenes on different levels of granularity. The granularity of a description is the context-dependent selection of an adequate level of detail in the description (Hobbs, 1985). Granularity plays a key role in human strategies to deal with the complexity of the spatial features of the real world. This is demonstrated nicely by an example from Hobbs (1985). In his example he points out that humans conceptualize streets as one-dimensional entities when they plan a trip, they use a two-dimensional conception when they cross a street. And in contexts where the pavement has to be dug up the street becomes a three-dimensional volume. The key importance of mechanisms to flexibly switch and translate between granularities for successful reasoning about the world is highlighted by the following quote from Hobbs (1985, p. 432):

Our ability to conceptualize the world at different granularities and to switch among these granularities is fundamental to our intelligence and flexibility. It enables us to map the complexities of the world around us into simple theories that are computationally tractable to reason in.

Imagine a scenario involving ships and their relative positions in the open sea (see Fig. 7). Ships can be modelled as elongated, directed entities neglecting their width or any other shape property. The resulting $\mathcal{D} \mathcal{R} \mathcal{A}_{f p}$ representation uses a single dipole for each ship to 
be represented (see left part of Fig. 7). In the $\mathcal{O P} \mathcal{R} \mathcal{A}_{1}^{*}$ representation in addition even the lengths of the ships are neglected (see middle part of Fig. 7). The $\mathcal{C} \mathcal{Y} \mathcal{C}_{b}$ representation abstracts away the different locations of the ships and only focuses on their relative orientation (see right part of Fig. 7).

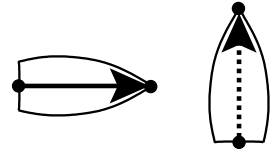

abstraction from shape

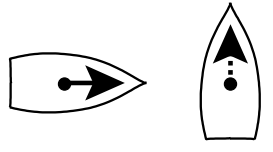

abstraction from length

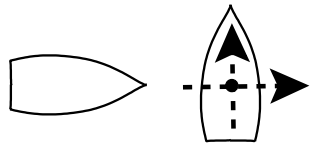

abstraction from location

Figure 7: Modelling relative ship directions on different levels of granularity with $\mathcal{D} \mathcal{R} \mathcal{A}_{f p}$, $\mathcal{O P} \mathcal{R} \mathcal{A}_{1}^{*}$, and $\mathcal{C Y \mathcal { C }}$.

In another example ships are represented with $\mathcal{D} \mathcal{R} \mathcal{A}_{f p}$ in such a way that the start point corresponds to the position of the ship and the end point represents its current speed. More specifically, the end point denotes the future position after one minute travel (if speed and heading were constant). Then longer arrows represent faster ships in a diagram. When we have an alternative representation in $\mathcal{O P} \mathcal{R} \mathcal{A}_{1}^{*}$, in this representation we might only focus on location and heading of the ships and abstract away from the their speed. Then several $\mathcal{D} \mathcal{R} \mathcal{A}_{f p}$ relations in one representation map onto a single $\mathcal{O P} \mathcal{R} \mathcal{A}_{1}^{*}$ relation in the alternative representation. For example the three relations \{fll, ells, illr\} are mapped to FRONTleft (see Fig. 8).

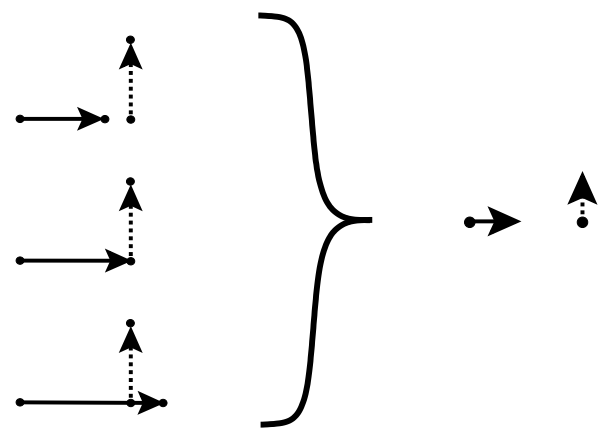

Figure 8: In a quotient homomorphism between $\mathcal{D} \mathcal{R} \mathcal{A}_{f p}$ and $\mathcal{O} \mathcal{P} \mathcal{R} \mathcal{A}_{1}^{*}$ the three relations \{fll, ells, illr\} are mapped to FRONTleft.

If different spatial calculi can be used to represent a given spatial situation at different levels of granularity, the relation between the calculi can typically be formalized as a quotient homomorphism. Figure 8 exemplifies the action of a quotient homomorphism. Homomorphisms also arise in other contexts, e.g. as embeddings of a smaller calculus into a larger 
one (for example, Allen's interval algebra can be embedded into $\mathcal{D} \mathcal{R} \mathcal{A}_{f p}$, see Proposition 25 below).

We now study homomorphisms in general. They are a means for the examination of relationships among calculi. Often, conceptual relations between different calculi and their domains can be formalised as homomorphism, and vice versa, if one has found a homomorphism, then often there is also some conceptual relation behind it.

Homomorphisms can also be used to transfer properties (like strength of composition, or algebraic closure deciding consistency) from one calculus to another one, see Propositions 16, 19, 23, 39, 40, 44, 46, 47 and 48 below. Using homomorphisms, it is also possible to find errors in composition tables (we discovered errors in 197 entries of the composition table of $\mathcal{O P} \mathcal{R} \mathcal{A}_{1}^{*}$, see Example 38 below).

Homomorphisms have been studied by Ligozat and Renz (2004) and Ligozat (2005, 2011) (mainly under the name of representations). We here introduce a more systematic treatment of homomorphisms. For non-associative algebras, we recall and refine the weaker notion of lax homomorphisms, which allow for both the embedding of a calculus into its domain, as well as relating several calculi to each other.

Definition 10 (Lax homomorphism, Moratz et al., 2009; Lücke, 2012). Given non-associative algebras $A$ and $B$, a lax homomorphism is a homomorphism $h: A \longrightarrow B$ on the underlying Boolean algebras such that:

- $h\left(\Delta_{A}\right) \geq \Delta_{B}$

- $h\left(a^{\smile}\right)=h(a)^{\smile}$ for all $a \in A$

- $h(a \diamond b) \geq h(a) \diamond h(b)$ for all $a, b \in A$

A lax homomorphism between complete atomic non-associative algebras is called semistrong (Mossakowski, Schröder, \& Wölfl, 2006) if for atoms $a, b$

$$
a \diamond b=\bigvee\{c \mid(h(a) \diamond h(b)) \wedge h(c) \neq 0\}
$$

This notion has been inspired by the definition of weak composition and will be used for representation homomorphisms of qualitative calculi.

Dually to lax homomorphisms, we can define oplax homomorphisms ${ }^{6}$, which enable us to define projections from one calculus to another.

Definition 11 (Oplax homomorphism, Moratz et al., 2009; Lücke, 2012). Given nonassociative algebras $A$ and $B$, an oplax homomorphism is a homomorphism $h: A \longrightarrow B$ on the underlying Boolean algebras such that:

- $h\left(\Delta_{A}\right) \leq \Delta_{B}$

- $h\left(a^{\smile}\right)=h(a)^{\smile}$ for all $a \in A$

- $h(a \diamond b) \leq h(a) \diamond h(b)$ for all $a, b \in A$

6. The terminology is motivated by that for monoidal functors. 
For quotients, we now introduce a strengthening of the notion of oplax homomorphism. A full ${ }^{7}$ homomorphism is an oplax homomorphism for which even

$$
h(a) \diamond h(b)=\bigvee_{h(a)=h(c), h(b)=h(d)} h(c \diamond d)
$$

A proper homomorphism (sometimes just called a homomorphism) of non-associative algebras is a homomorphism that is lax and oplax at the same time; the above inequalities then turn into equations. Each proper homomorphism is also full. A proper injective homomorphism is also semi-strong.

A homomorphism between complete atomic non-associative algebras can be given by its action on base-relations; it is extended to general relations by

$$
h\left(\bigvee_{i \in I} b_{i}\right)=\bigvee_{i \in I} h\left(b_{i}\right)
$$

where $\bigvee$ is arbitrary (possibly infinite) join. In the sequel, we will always define homomorphisms in this way.

While semi-strong lax homomorphisms can be used to transfer the composition from the target to the source algebra, surjective full oplax homomorphisms can be used for a transfer in the opposite direction. We now study the latter, the former will be treated in Def. 20.

Definition 12. Given a complete atomic non-associative algebra $A$ and an equivalence relation $\sim$ on the atoms of $A$ that is a congruence for ${ }_{-}{ }^{-}$, we define the quotient algebra $A / \sim_{A}$ to have equivalence classes of $A$-atoms as atoms. General relations are then sets of such atoms. We further define for atoms $a, b$ :

$$
\begin{aligned}
& \Delta_{A / \sim}=\left\{[a] \mid a \in \Delta_{A}\right\} \\
& {[a]^{-}=\left[a^{\smile}\right]} \\
& {[a] \diamond[b]=\left\{[c] \mid c \in a^{\prime} \diamond b^{\prime}, a^{\prime} \sim a, b^{\prime} \sim b\right\}}
\end{aligned}
$$

where as usual we treat general relations as sets of atoms; hence general relations in $A / \sim_{A}$ are sets of equivalence classes of $A$-atoms.

Unfortunately, in general, $A / \sim_{A}$ will not be a non-associative algebra again:

Example 13. Consider the relation algebra of the $\mathcal{C Y C}_{b}$ calculus (Example 6) and the equivalence relation generated by $o \sim e$. The quotient algebra fails to satisfy the identity laws (laws (b) in Def. 1). This can be seen from the quotient composition table:

\begin{tabular}{|c||c|c|c|}
\hline$\circ$ & $\{e, o\}$ & $\{l\}$ & $\{r\}$ \\
\hline \hline$\{e, o\}$ & $\{e, o\}$ & $\{\{l\},\{r\}\}$ & $\{\{l\},\{r\}\}$ \\
\hline$\{l\}$ & $\{\{l\},\{r\}\}$ & $\{\{e, o\},\{l\},\{r\}\}$ & $\{\{e, o\},\{l\},\{r\}\}$ \\
\hline$\{r\}$ & $\{\{l\},\{r\}\}$ & $\{\{e, o\},\{l\},\{r\}\}$ & $\{\{e, o\},\{l\},\{r\}\}$ \\
\hline
\end{tabular}

7. This terminology is borrowed from the theory of partial algebras (Burmeister, 1986). Burmeister (2002, p. 101) puts it as follows: " $f$ is full iff $f$ fully induces the structure on its direct image $f(A)$." — which is exactly what he want here, too. 
We will study a method to prove that $A / \sim_{A}$ is a non-associative algebra later under additional conditions. For now, it is straightforward to prove:

Proposition 14. If the algebra $A / \sim_{A}$ defined in Def. 12 is a non-associative algebra, the homomorphism $q: A \rightarrow A / \sim_{A}$ given by $a \mapsto[a]$ is surjective and full.

This naturally leads to:

Definition 15. An oplax homomorphism of non-associative algebras is said to be a quotient homomorphism if it is full and surjective.

An easy standard result from universal algebra (Grätzer, 1979) gives us:

Proposition 16. Proper quotient homomorphisms preserve the holding of equations, in particular, associativity.

However, non-proper quotient homomorphisms in general do not preserve the holding of equations. See Example 35: $\mathcal{D} \mathcal{R} \mathcal{A}_{f p}$ is associative, but its quotient $\mathcal{D} \mathcal{R} \mathcal{A}_{f}$ is not.

This raises the question why we do not use the standard constructions and results of universal algebra (Grätzer, 1979; Maddux, 2006), where homomorphisms are always proper and hence quotients preserve equations (Prop. 16) and thus the quotient of a non-associative algebra is a non-associative algebra again. The reason is the following:

Example 17. Consider the point algebra induced by the three base-relations $<,=$ and $>$, with converse and composition tables:

\begin{tabular}{|l||l|}
\hline$a$ & $a$ \\
\hline \hline$<$ & $>$ \\
\hline$=$ & $=$ \\
\hline$>$ & $<$ \\
\hline
\end{tabular}

\begin{tabular}{|c||c|c|c|}
\hline$\circ$ & $<$ & $=$ & $>$ \\
\hline \hline$<$ & $<$ & $<$ & $\{<,=,>\}$ \\
\hline$=$ & $<$ & $=$ & $>$ \\
\hline$>$ & $\{<,=,>\}$ & $>$ & $>$ \\
\hline
\end{tabular}

Let $\sim$ be the standard algebraic congruence relation generated by $\langle\sim\rangle$. Then $<$ is equal to $<\diamond<$, which is congruent to $\langle\diamond>$, which is $\{<,=,>\}$. Similarly, $>$ is congruent to $\{<,=,>\}$. Since congruence respects meet, we obtain that $\langle\cap\rangle$, which is $\emptyset$, is congruent to $\{<,=,>\}$. This means that the congruence is trivial and the standard algebraic quotient is the trivial one-point relation algebra.

By contrast, with our notion of quotient, we obtain the following relation algebra, which is the expected one (we denote the equivalence class $\{<,>\}$ by $\neq$ ):

\begin{tabular}{|l||l|}
\hline$a$ & $a^{\smile}$ \\
\hline \hline$\neq$ & $\neq$ \\
\hline$=$ & $=$ \\
\hline
\end{tabular}

\begin{tabular}{|c||c|c|}
\hline$\circ$ & $\neq$ & $=$ \\
\hline \hline$\neq$ & $\{\neq,=\}$ & $\neq$ \\
\hline$=$ & $\neq$ & $=$ \\
\hline
\end{tabular}

The corresponding quotient homomorphism is not proper: $q(<) \diamond q(<)$ is $\neq \diamond \neq$, which is $\{\neq,=\}$, but $q(<\diamond<)=q(<)$, which is $\neq$. However, by Prop. 14, it is surjective and full.

Proposition 18. In the context of Prop. 14, if $q$ is proper, then $A / \sim_{A}$ is a non-associative algebra. 
Proof. By Prop. 16, we know that equations are preserved by $q$. The only axiom in Def. 1 not in equational form is (g). Now Tarski has shown (Maddux, 2006) that (g) is equivalent to

$$
\left(a^{\smile} \diamond(1-(a \diamond b))\right) \vee(1-b)=1-b
$$

An important application of quotients and quotient homomorphisms lies in the following fact:

Proposition 19. Given a quotient homomorphism $q: A \rightarrow B, B$ 's converse and composition tables can be computed from those for $A$, using $q$.

Proof. Use the formulas for converse resp. composition from the definition of full homomorphism. Since $q$ is surjective, the formulas work for all elements of $B$.

Another important application of homomorphisms is their use in the definition of a qualitative calculus. Ligozat and Renz (2004) define a qualitative calculus in terms of a so-called weak representation (Ligozat, 2005, 2011):

Definition 20 (Weak representation). A weak representation $\varphi: A \rightarrow \mathcal{P}(\mathcal{U} \times \mathcal{U})^{8}$ is an identity-preserving (i.e. $\varphi\left(\Delta_{A}\right)=\Delta_{B}$ ) and converse-preserving lax homomorphism $\varphi$ from a complete atomic non-associative algebra $A$ into the relation algebra of a domain $\mathcal{U}$. The latter is given by the canonical relation algebra on the powerset $\mathcal{P}(\mathcal{U} \times \mathcal{U})$, where identity, converse and composition (as well as the Boolean algebra operations) are given by their set-theoretic interpretations. A weak representation is semi-strong if $\varphi$ is semi-strong. It is strong, if $\varphi$ is strong.

Example 21. Let $\mathbb{D}=\left\{(s, e) \mid s, e \in \mathbb{R}^{2}, s \neq e\right\}$ be the set of all dipoles in $\mathbb{R}^{2}$. Then the weak representation of $\mathcal{D} \mathcal{R} \mathcal{A}_{f p}$ is the lax homomorphism $\varphi_{f}: \mathcal{D} \mathcal{R} \mathcal{A}_{f p} \longrightarrow \mathcal{P}(\mathbb{D} \times \mathbb{D})$ given by

$$
\varphi_{f}(b)=b .
$$

Here, the $b$ on the left hand-side of the equation is an element of the abstract relation algebra, while the $b$ on the right hand-side is the set-theoretic extension as a relation. Since we have chosen to use set-theoretic relations themselves as elements of the relation algebra, here both are the same.

This can be generalized as follows:

Proposition 22. Semi-strong representations and partition schemes are in one-one correspondence.

Proof. Given a partition scheme, by Prop. 4, we obtain a non-associative algebra. Let $\varphi$ map each general relation $R \subseteq \mathcal{P}(I)$ to $\bigcup_{i \in R} R_{i}$. The definition of weak composition ensures that $\varphi$ is a semi-strong lax homomorphism. Conversely, given a semi-strong representation $\varphi: A \rightarrow \mathcal{P}(\mathcal{U} \times \mathcal{U})$, define a partition scheme on the atoms of $A$ by putting $R_{a}:=\varphi(a)$.

8. Note that the domain and codomain are part of the weak representation. 
Preservation of top, bottom and meet by $\varphi$ ensure the JEPD property. Moreover, by semistrength, composition in $A$ is just weak composition according to the partition scheme. It is clear that these constructions are inverses of each other.

The following propositions are straightforward.

Proposition 23 (Moratz et al., 2009; Lücke, 2012). A calculus has strong composition if and only if its weak representation is a proper homomorphism.

Proposition 24 (Ligozat, 2005). A weak representation $\varphi$ is injective if and only if $\varphi(b) \neq \emptyset$ for each base-relation $b$.

A first sample use of homomorphism is the embedding of Allen's interval relations (Allen, 1983) into $\mathcal{D} \mathcal{R} \mathcal{A}_{f p}$ via a homomorphism.

Proposition 25 (Moratz et al., 2011). A proper homomorphism from Allen's interval algebra to $\mathcal{D} \mathcal{R} \mathcal{A}_{f p}$ exists and is given by the following mapping of base-relations.

\begin{tabular}{|c|c|c|c|c|}
\hline equals & $\mapsto$ & sese & & \\
\hline before & $\mapsto$ & $\mathrm{ffbb}$ & before $\smile$ & $\mapsto$ \\
\hline meets & $\mapsto$ & efbs & meets $^{\smile}$ & $\mapsto$ \\
\hline overlaps & $\mapsto$ & ifbi & overlaps $\smile$ & $\mapsto$ \\
\hline during & $\mapsto$ & bfii & during $\smile$ & $\mapsto$ \\
\hline starts & $\mapsto$ & sfsi & starts $\smile$ & $\mapsto$ \\
\hline finishes & $\mapsto$ & beie & finishes $\smile$ & $\mapsto$ \\
\hline
\end{tabular}

When studying quotients of calculi, it is natural to consider homomorphisms of weak representations. We refine the notion by Moratz et al. (2009) and Lücke (2012) in order to fit it better to the examples:

Definition 26. Given weak representations $\varphi: A \rightarrow \mathcal{P}(\mathcal{U} \times \mathcal{U})$ and $\psi: B \rightarrow \mathcal{P}(\mathcal{V} \times \mathcal{V})$, $a \in\{$ lax, oplax, full, proper $\}$ and $b \in\{$ lax, oplax, proper $\}$, an $(a, b)$-homomorphism of weak representations $(h, i): \varphi \rightarrow \psi$ is given by

- an $a$-homomorphism of non-associative algebras $h: A \rightarrow B$, and

- a map $i: \mathcal{U} \rightarrow \mathcal{V}$, such that the diagram

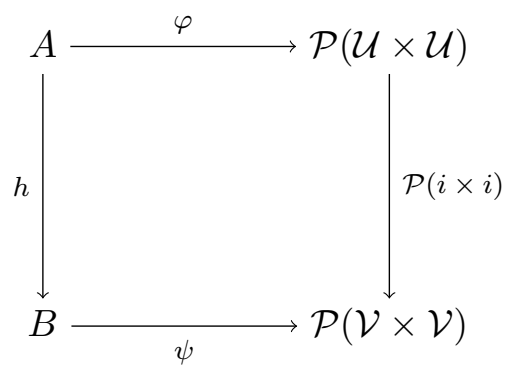


commutes according to $b$. Here, lax commutation means that for all $R \in A, \psi(h(R)) \subseteq$ $\mathcal{P}(i \times i)(\varphi(R))$, oplax commutation means the same with $\supseteq$, and proper commutation with $=$. Note that $\mathcal{P}(i \times i)$ is the obvious extension of $i$ to a function between relation algebras; further note that (unless $i$ is bijective) this is not even a homomorphism of Boolean algebras (it may fail to preserve top, intersections and complements), although it satisfies the oplaxness property (and the laxness property if $i$ is injective) ${ }^{9}$.

Ligozat (2005) defines a more special notion of morphism between weak representations; it corresponds to our notion of (proper,oplax) homomorphism of weak representations where the component $h$ is the identity.

Example 27. The homomorphism from Prop. 25 can be extended to a (proper, proper) homomorphism of weak representations by letting $i$ be the embedding of time intervals to dipoles on the $x$-axis.

Definition 28. A quotient homomorphism of weak representations is a (full,oplax) homomorphism of weak representations that is surjective in both components.

We also refine the construction of a weak representation from an equivalence relation on the domain introduced by Moratz et al. (2009) and Lücke (2012), whose constructions in typical cases will produce a trivial one-point quotient, cf. Example 17.

Definition 29. Given a weak representation $\varphi: A \rightarrow \mathcal{P}(\mathcal{U} \times \mathcal{U})$ and an equivalence relation $\sim$ on $\mathcal{U}$ that is a congruence for $\_\_$, we obtain the quotient representation $\varphi / \sim$ as follows:

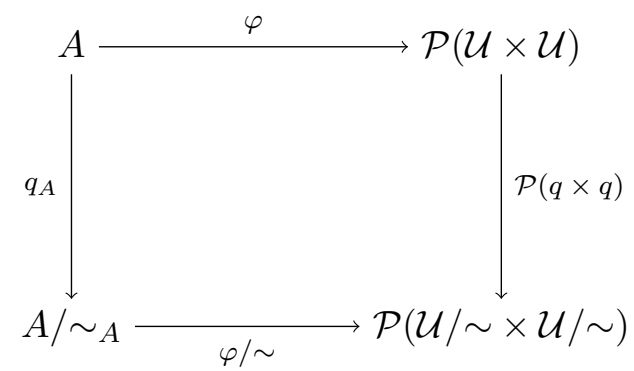

- Let $q: \mathcal{U} \rightarrow \mathcal{U} / \sim$ be the set-theoretic factorization of $\mathcal{U}$ by $\sim$;

- $q$ extends to relations: $\mathcal{P}(q \times q): \mathcal{P}(\mathcal{U} \times \mathcal{U}) \rightarrow \mathcal{P}(\mathcal{U} / \sim \times \mathcal{U} / \sim)$;

- let $\sim_{A}$ be the equivalence relation on the atoms of $A$ generated by

$$
\mathcal{P}(q \times q)\left(\varphi\left(b_{1}\right)\right) \cap \mathcal{P}(q \times q)\left(\varphi\left(b_{2}\right)\right) \neq \emptyset \Rightarrow b_{1} \sim_{A} b_{2}
$$

for base-relations $b_{1}, b_{2} \in A$;

- let $q_{A}: A \rightarrow A / \sim_{A}$ be the quotient of $A$ by $\sim_{A}$ in the sense of Def. 12 ;

9. The reader with background in category theory may notice that the categorically more natural formulation would use the contravariant powerset functor, which yields homomorphisms of Boolean algebras (Mossakowski et al., 2006). However, the present formulation fits better with the examples. 
- finally, the function $\varphi / \sim$ is defined as

$$
\varphi / \sim(R)=\mathcal{P}(q \times q)\left(\varphi\left(q_{A}^{-1}(R)\right)\right) .
$$

$\sim$ is called regular w.r.t. $\varphi$ if $\sim_{A}$ is the kernel of $\mathcal{P}(q \times q) \circ \varphi$ (i.e. the set of all pairs made equal by $\mathcal{P}(q \times q) \circ \varphi)$. In this case, each base-relation $b \in A$ already generates (via $\mathcal{P}(q \times q) \circ \varphi)$ the full relation of the equivalence class $[b] \in A / \sim_{A}$.

Proposition 30. Let a strong representation $\varphi: A \rightarrow \mathcal{P}(\mathcal{U} \times \mathcal{U})$ of a complete atomic nonassociative algebra $A$ and an equivalence relation $\sim$ on $\mathcal{U}$ be given, such that $\sim$

1. is identity-regular, that is $(\varphi(a) \cap \sim) \neq \emptyset$ implies $\varphi(a) \subseteq \sim$

2. is a congruence for converse, and

3. enjoys the following fill-in property: if $u \varphi(a) x$ and $u \sim y$, then there exist $a^{\prime} \sim a$ and $z \sim x$ with

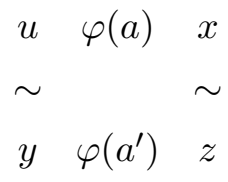

Then $A / \sim_{A}$ as defined in Def. 29 is a non-associative algebra, $q_{A}: A \rightarrow A / \sim_{A}$ is a quotient homomorphism, and $\varphi / \sim$ is a semi-strong lax homomorphism of non-associative algebras.

Proof. We use the atoms of $A / \sim_{A}$ to define a partition scheme $b \in A t\left(A / \sim_{A}\right) \mapsto \varphi / \sim(b)$. Note that we know that $A / \sim_{A}$ is a Boolean algebra (although we do not know yet that it is a non-associative algebra). It is straightforward to show that $\varphi / \sim$ preserves bottom and joins; since $q$ is surjective, also top is preserved. Concerning meets, since general relations in $A / \sim_{A}$ can be considered to be sets of base-relations, it suffices to show that $b_{1} \wedge b_{2}=0$ implies $\mathcal{P}(q \times q)\left(\varphi\left(q_{A}^{-1}\left(b_{1}\right)\right)\right) \cap \mathcal{P}(q \times q)\left(\varphi\left(q_{A}^{-1}\left(b_{2}\right)\right)\right)=\emptyset$. Assume to the contrary that $\mathcal{P}(q \times$ $q)\left(\varphi\left(q_{A}^{-1}\left(b_{1}\right)\right)\right) \cap \mathcal{P}(q \times q)\left(\varphi\left(q_{A}^{-1}\left(b_{2}\right)\right)\right) \neq \emptyset$. Then already $\mathcal{P}(q \times q)\left(\varphi\left(b_{1}^{\prime}\right)\right) \cap \mathcal{P}(q \times q)\left(\varphi\left(b_{2}^{\prime}\right)\right) \neq \emptyset$ for base-relations $b_{i}^{\prime} \in q_{A}^{-1}\left(b_{i}\right), i=1,2$. But then $b_{1}^{\prime} \sim_{A} b_{2}^{\prime}$, hence $q_{A}\left(b_{1}^{\prime}\right)=q_{A}\left(b_{2}^{\prime}\right) \leq b_{1} \wedge b_{2}$, contradicting $b_{1} \wedge b_{2}=0$. From these preservation properties, the JEPD property follows. By identity-regularity and converse being a congruence, the condition of a partition scheme on identity and converse are fulfilled.

By Prop. 22, we obtain a semi-strong representation $\varphi / \sim: B \rightarrow \mathcal{P}(\mathcal{U} / \sim \times \mathcal{U} / \sim)$. In order to show that $A / \sim_{A}$ is a non-associative algebra, we show that $A / \sim_{A}=B$. We already know that they have the same atoms and thus agree as complete atomic Boolean algebras. We show agreement on the remaining operations:

Identity: Since $\varphi$ is identity-preserving, $\Delta_{A}$ is atomic. By identity-regularity, $\Delta_{B}=\left[\Delta_{A}\right]=$ $\Delta_{A / \sim_{A}}$

Converse: Since $\sim$ is a congruence for converse, for an atomic relation $a \in A,[a]^{\smile_{B}}=$ $\left[a^{\smile A}\right]=[a]^{\smile A / \sim A}$. 
Composition: Given atomic relations $a, b \in A$, we have $[c] \in[a] \diamond_{B}[b]$ iff (by definition of weak composition) there exist $x, y, z$ with $[x] \varphi / \sim(a)[y] \varphi / \sim(b)[z]$ and $[x] \varphi / \sim(c)[z]$ iff (by definition of $\varphi / \sim$ ) there exist $x_{1}, x_{2}, y_{1}, x_{2}, z_{1}, z_{2}$ and $a^{\prime} \sim a, b^{\prime} \sim b, c^{\prime} \sim c$ with

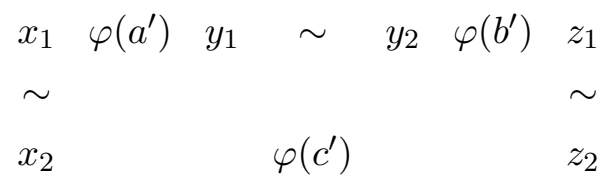

By the fill-in property, this is equivalent to (implicitly quantifying variables existentially and omitting $\left.a^{\prime} \sim a, b^{\prime} \sim b, c^{\prime} \sim c\right)$ :

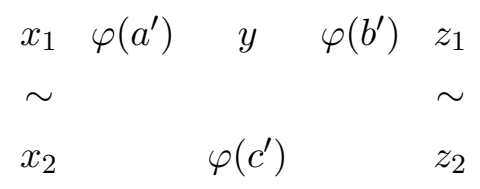

By strength of $\varphi$, this is equivalent to

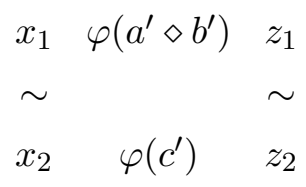

which in turn is equivalent to $c^{\prime} \sim c^{\prime \prime} \in\left(a^{\prime} \diamond b^{\prime}\right)$ (for some $c^{\prime \prime}$ and $a^{\prime} \sim a, b^{\prime} \sim b, c^{\prime} \sim c$ ). Now this is equivalent to $[c] \in[a] \diamond_{A / \sim_{A}}[b]$.

This completes the proof that $A / \sim_{A}$ is a non-associative algebra. By Prop. 14, $q_{A}: A \rightarrow$ $A / \sim_{A}$ is a quotient homomorphism, and by Prop. $22, \varphi / \sim$ is a semi-strong lax homomorphism.

An interesting open question is whether Prop. 30 also holds for semi-strong representations. We conjecture that the answer is positive. Note that $\varphi / \sim$ can fail to be strong even if $\varphi$ is (consider the quotient $\mathcal{D} \mathcal{R} \mathcal{A}_{f}$ of $\mathcal{D} \mathcal{R} \mathcal{A}_{f p}$ introduced in Example 35).

Example 31. $\mathcal{C} \mathcal{Y} \mathcal{C}_{b}$ is a quotient of $\mathcal{O P} \mathcal{R} \mathcal{A}_{1}^{*}$. At the level of domains, it acts as follows: an oriented point $(p, \phi)$ is mapped to the orientation $\phi$ (the point $p$ is forgotten). At the level of non-associative algebras, the quotient is given by the table in Fig. 9.

Proposition 32. Under the conditions of Prop. 30, $\left(q_{A}, q\right): \varphi \rightarrow \varphi / \sim$ is a (full, oplax) quotient homomorphism of semi-strong representations. If $\sim$ is regular w.r.t. $\varphi$, then $\left(q_{A}, q\right)$ is (full,proper) and satisfies the following universal property: if ( $q_{B}: A \rightarrow B, i: \mathcal{U} \rightarrow$ $\mathcal{V}): \varphi \rightarrow \psi$ is another (full,proper) homomorphism of weak representations with $\psi$ injective and $\sim \subseteq \operatorname{ker}(i)$, then there is a unique (full,proper) homomorphism of weak representations $(h, k): \varphi / \sim \rightarrow \psi$ with $\left(q_{B}, i\right)=(h, k) \circ\left(q_{A}, q\right)$. 


\begin{abstract}
\{LEFTleftA, FRONTfront, BACKback, RIGHTrightA, SAMEback\} $\mapsto \mathrm{o}$
$\{$ LEFTleft+, LEFTback, LEFTright+, RIGHTright+, RIGHTleft+, RIGHTfront, FRONTleft, BACKright, SAMEleft $\} \mapsto 1$

\{LEFTleft-, LEFTfront, LEFTright-, RIGHTright-, RIGHTleft-,

RIGHTback, FRONTright, BACKleft, SAMEright $\} \mapsto r$

\{LEFTrightP, RIGHTleftP, FRONTback, BACKfront, SAMEfront $\} \mapsto \mathrm{e}$
\end{abstract}

Figure 9: Mapping from $\mathcal{O P} \mathcal{R} \mathcal{A}_{1}^{*}$ to $\mathcal{C} \mathcal{Y} \mathcal{C}_{b}$ relations

Proof. The (full,_) property ${ }^{10}$ follows from Prop. 14. The (_,oplax) property for $\left(q_{A}, q\right)$ is $\mathcal{P}(q \times q) \circ \varphi \subseteq \varphi / \sim \circ q_{A}$, which by definition of $\varphi / \sim$ amounts to

$$
\mathcal{P}(q \times q) \circ \varphi \subseteq \mathcal{P}(q \times q) \circ \varphi \circ q_{A}^{-1} \circ q_{A},
$$

which follows from surjectivity of $q$. Regularity of $\sim$ w.r.t. $\varphi$ means that $\sim_{A}$ is the kernel of $\mathcal{P}(q \times q) \circ \varphi$, which turns the above inequation into an equality; hence we obtain (_,properness). Concerning the universal property, let $\left(q_{B}, i\right): \varphi \rightarrow \psi$ with the mentioned properties be given. Since $\sim \subseteq \operatorname{ker}(i)$, there is a unique function $k: \mathcal{U} / \sim \rightarrow \mathcal{V}$ with $i=k \circ q$. The homomorphism $h$ we are looking for is determined uniquely by $h\left(q_{A}(b)\right)=q_{B}(b)$; this also ensures the (full,proper) homomorphism property. All that remains to be shown is well-definedness. Suppose that $b_{1} \sim_{A} b_{2}$. By regularity, $\mathcal{P}(q \times q)\left(\varphi\left(b_{1}\right)\right)=\mathcal{P}(q \times q)\left(\varphi\left(b_{2}\right)\right)$. Hence also $\mathcal{P}(i \times i)\left(\varphi\left(b_{1}\right)\right)=\mathcal{P}(i \times i)\left(\varphi\left(b_{2}\right)\right)$ and $\psi\left(q_{B}\left(b_{1}\right)\right)=\psi\left(q_{B}\left(b_{2}\right)\right)$. By injectivity of $\psi$, we get $q_{B}\left(b_{1}\right)=q_{B}\left(b_{2}\right)$.

Example 33. The equivalence relation of the quotient in Ex. 31 is regular, and consequently, the quotient of weak representations is (full,proper), cf. Fig. 10 illustrating this for the relation RIGHTright + .

So far, we have studied quotients arising from quotienting the domain. There are also quotients leaving the domain intact and just identifying certain base-relations.

Proposition 34. Let $\varphi: A \rightarrow \mathcal{P}(\mathcal{U} \times \mathcal{U})$ be a semi-strong representation of a complete atomic non-associative algebra $A$ and $\sim_{A}^{11}$ be an equivalence relation on the atoms (base-relations) of $A$ that does not relate $\Delta$ with any other relation and that is a congruence for _ $~$. This leads to a (full, oplax) quotient of the weak representation as follows:

10. We write _ as a placeholder for "don't care", i.e. (full,_) only refers to fullness of the first component. 11. Note that in contrast to Def. 29 , where $\sim_{A}$ is constructed, here $\sim_{A}$ is a parameter that can be chosen. 


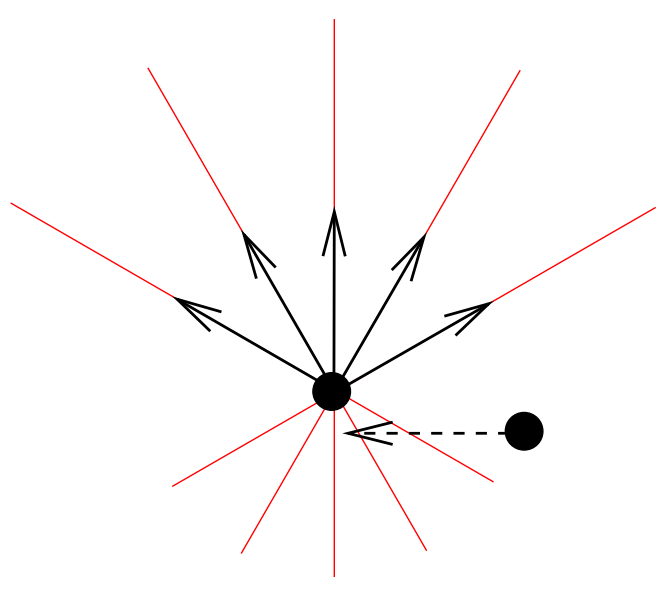

Figure 10: The $\mathcal{O P} \mathcal{R} \mathcal{A}_{1}^{*}$ relation RIGHTright + generates all possible angles for the $\mathcal{C Y} \mathcal{C}_{b}$ relation $\mathrm{r}$.

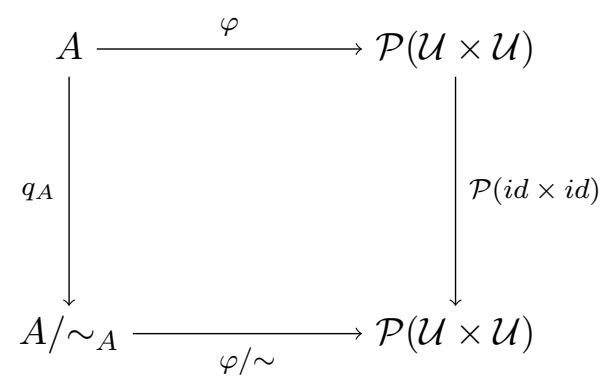

Proof. Let $q_{A}: A \rightarrow A / \sim_{A}$ be defined as in Def. 12. Then $\varphi / \sim$ can be defined similarly as in Def. 29 (where $q=i d$ ). From this, we get a semi-strong representation $\varphi / \sim: B \rightarrow$ $\mathcal{P}(\mathcal{U} \times \mathcal{U})$ as in Prop. 30. The proof of $A / \sim_{A}=B$ parallels that in Prop. 30 for the Boolean algebra structure and for converse. For identity, we use the assumption about $\Delta$, which implies that $\Delta_{A / \sim_{A}}=\left\{\Delta_{A}\right\}$. Concerning composition, we need semi-strength only: $[c] \in[a] \diamond_{B}[b]$ iff there exist $x, y, z$ with $x \varphi / \sim(a) y \varphi / \sim(b) z$ and $x \varphi / \sim(c) z$ iff there exist $x, y, z$ and $a^{\prime} \sim a, b^{\prime} \sim b, c^{\prime} \sim c$ with $x a^{\prime} y b^{\prime} z$ and $x c^{\prime} z$ iff (by semi-strength) there exist $a^{\prime} \sim a, b^{\prime} \sim b, c^{\prime} \sim c$ with $c^{\prime} \in a^{\prime} \diamond b^{\prime}$ iff $[c] \in[a] \diamond_{A / \sim_{A}}[b]$.

The (full, oplax)-property follows from Prop. 14 and as in Prop. 32.

Example 35. $\mathcal{D} \mathcal{R} \mathcal{A}_{f}$ (as a semi-strong representation) is a quotient of $\mathcal{D} \mathcal{R} \mathcal{A}_{f p}$. It is obtained by forgetting the labels '+', '-', 'P' and 'A'.

Example 36. $\mathcal{O P} \mathcal{R} \mathcal{A}_{n}$ is a quotient of $\mathcal{O P} \mathcal{R} \mathcal{A}_{n \cdot m}$, which is the identity at the domain level. At the level of non-associative algebras, $q_{A}$ maps region $i \cdot m$ in $\mathcal{O P} \mathcal{R} \mathcal{A}_{n \cdot m}$ to region $i$ in $\mathcal{O P R} \mathcal{A}_{n}$ (for even $i$ ), and regions $(i-1) \cdot m+1$ to $(i+1) \cdot m-1$ in $\mathcal{O P} \mathcal{R} \mathcal{A}_{n \cdot m}$ to region $i$ in $\mathcal{O P} \mathcal{R} \mathcal{A}_{n}$ (for odd $i$ ), see Fig. 11. This is canonically extended the $\mathcal{O P} \mathcal{R} \mathcal{A}$ relations. The equivalence relation $\sim_{A}$ is the kernel of $q_{A}$, i.e. it relates $i \cdot m$ only to itself, while all elements from $(i-1) \cdot m+1$ to $(i+1) \cdot m-1$ are related to each other. 
Note that this yields an oplax homomorphism of non-associative algebra that is not lax. A counterexample to laxness of $\mathcal{O P} \mathcal{R} \mathcal{A}_{2} \rightarrow \mathcal{O P} \mathcal{R} \mathcal{A}_{1}$ is the following: $h\left({ }_{2} \angle_{0}^{0} \diamond_{2} \angle_{2}^{1}\right)=\left\{{ }_{1} \angle_{3}^{1}\right\}$, but $h\left({ }_{2} \angle_{0}^{0}\right) \diamond h\left({ }_{2} \angle_{2}^{1}\right)=\left\{{ }_{1} \angle_{3}^{1},{ }_{1} \angle_{3}^{2},{ }_{1} \angle_{3}^{3}\right\}$.
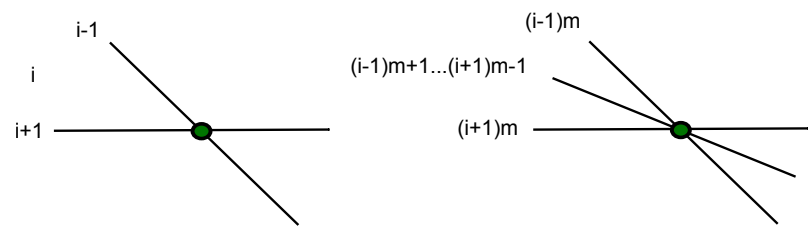

Figure 11: $\mathcal{O P} \mathcal{R} \mathcal{A}_{n}$ is a quotient of $\mathcal{O P} \mathcal{R} \mathcal{A}_{m \cdot n}$

In (Dylla, Mossakowski, Schneider, \& Wolter, 2013), we show that $\mathcal{O P} \mathcal{R} \mathcal{A}_{1}$ to $\mathcal{O P} \mathcal{R} \mathcal{A}_{8}$ are not associative. By Prop. 16 and Ex. 36, this carries over to any $\mathcal{O P \mathcal { R }} \mathcal{A}_{n}$.

Example 37. $\mathcal{O P} \mathcal{R} \mathcal{A}_{1}$ is a quotient of $\mathcal{O P} \mathcal{R} \mathcal{A}_{1}^{*}$. It is the identity at the domain level. At the level of non-associative algebras, it forgets the labels '+', '-', 'P' and 'A'. 


$$
\begin{aligned}
& \text { \{1ll1A }\} \mapsto \text { LEFTleftA } \\
& \{111 l+, \text { lllr, lllb }\} \mapsto \text { LEFTleft }+ \\
& \text { \{llll-, lrll, lbll }\} \mapsto \text { LEFTleft- } \\
& \text { \{ffff, eses, fefe, fifi, ibib, fbii, fsei, ebis, iifb, eifs, iseb }\} \mapsto \text { FRONTfront } \\
& \{\text { bbbb }\} \mapsto \text { BACKback } \\
& \{\text { llbr }\} \mapsto \text { LEFTback } \\
& \{\text { llfl, lril, lsel }\} \mapsto \text { LEFTfront } \\
& \text { \{llrrP }\} \mapsto \text { LEFTrightP } \\
& \{\text { llrr }+\} \mapsto \text { LEFTright }+ \\
& \{l l r f \text {, llrl, llrr-, lfrr, lrrr, lere, lirl, lrri, lrrl }\} \mapsto \text { LEFTright- } \\
& \{\text { rrrrA }\} \mapsto \text { RIGHTrightA } \\
& \text { \{rrrr+, rbrr, rlrr }\} \mapsto \text { RIGHTright+ } \\
& \text { \{rrr- }- \text { rrrl, rrrb }\} \mapsto \text { RIGHTright- } \\
& \{\text { rrllP }\} \mapsto \text { RIGHTleftP } \\
& \{\text { rrll }+ \text {, rrlr, rrlf, rlll, rfll, rllr, rele, rlli, rilr }\} \mapsto \text { RIGHTleft }+ \\
& \text { \{rrll- }\} \mapsto \text { RIGHTleft- } \\
& \{\text { rrbl }\} \mapsto \text { RIGHTback } \\
& \text { \{rrfr, rser, rlir\} } \mapsto \text { RIGHTfront } \\
& \text { \{ffbb, efbs, ifbi, iibf, iebe }\} \mapsto \text { FRONTback } \\
& \text { \{frrr, errs, irrl }\} \mapsto \text { FRONTright } \\
& \text { \{fll, ells, illr }\} \mapsto \text { FRONTleft } \\
& \text { \{blrr }\} \mapsto \text { BACKright } \\
& \{\text { brll }\} \mapsto \text { BACKleft } \\
& \text { \{bbff, bfii, beie, bsef, biif }\} \mapsto \text { BACKfront } \\
& \text { \{slsr }\} \mapsto \text { SAMEleft } \\
& \text { \{sese, sfsi, sisf }\} \mapsto \text { SAMEfront } \\
& \{\text { sbsb }\} \mapsto \text { SAMEback } \\
& \{\mathrm{srsl}\} \mapsto \text { SAMEright }
\end{aligned}
$$

Figure 12: Mapping from $\mathcal{D} \mathcal{R} \mathcal{A}_{f p}$ to $\mathcal{O P} \mathcal{R} \mathcal{A}_{1}^{*}$ relations

Example 38 (refined from Moratz et al. 2009; Lücke 2012). $\mathcal{O P} \mathcal{R} \mathcal{A}_{1}^{*}$ is a quotient of $\mathcal{D} \mathcal{R} \mathcal{A}_{f p}$. At the level of non-associative algebras, the quotient is given by the table in Fig. 12. At the level of domains, it acts as follows: Given dipoles $d_{1}, d_{2} \in \mathbb{D}$, the relation $d_{1} \sim d_{2}$ expresses that $d_{1}$ and $d_{2}$ have the same start point and point in the same direction. (This is regular w.r.t. $\varphi_{f}$.) Then $\mathbb{D} / \sim$ is the domain $\mathbb{O P}$ of oriented points in $\mathbb{R}^{2}$. See Fig. 14 . 
The equivalence relation of this quotient is indeed regular: given a base-relation $b \in \mathcal{D} \mathcal{R} \mathcal{A}_{f p}$, $\varphi(b)$ already generates (via the quotient) the whole of $\varphi / \sim([b])$ : for any pair of oriented points in $\varphi / \sim([b])$, a suitable choice of dipole end points leads to a relation in $\varphi(b)$ (also cf. Fig. 8). Consequently, the quotient of weak representations is (full,proper).

By Prop. 19, the construction of $\mathcal{O P} \mathcal{R} \mathcal{A}_{1}^{*}$ as a quotient allows us the computation of the converse and composition tables by applying the congruence relations to the tables for $\mathcal{D} \mathcal{R} \mathcal{A}_{f p}$. Actually, we have compared the result of this procedure with the composition table for $\mathcal{O P} \mathcal{R} \mathcal{A}_{1}^{*}$ published by Dylla (2008) and provided with the tool SparQ (Wallgrün, Frommberger, Dylla, \& Wolter, 2009). In the course of checking the full oplaxness property of the quotient homomorphism from $\mathcal{D} \mathcal{R} \mathcal{A}_{f p}$ to $\mathcal{O P \mathcal { R }} \mathcal{A}_{1}^{*}$, we discovered errors in 197 entries of the composition table of $\mathcal{O P} \mathcal{R} \mathcal{A}_{1}^{*}$ as it was shipped with the qualitative reasoner $\operatorname{SparQ}{ }^{12}$ The table has been corrected accordingly in the meantime. ${ }^{13}$

For example, the composition of SAMEright $=q(\mathrm{srsl})$ and RIGHTright $+=q(\mathrm{rrrr}+, \mathrm{rbrr}$, rlrr) can be computed as $q(\{$ blrr, lere, lfrr, lirl, llrf, llrl, llrr+, llrr-, llrrp, lrri, $\operatorname{lrrl}, \operatorname{lrrr}, \operatorname{rbrr}, \ln \operatorname{rr}, \operatorname{rrrr}+\})=\{$ LEFTright-, LEFTright + , LEFTrightP, BACKright, RIGHTright +$\}$. Now the old table additionally contained RIGHTright-. However, the configuration $a$ SAMEright $b, b$ RIGHTright $+c$ and $a$ RIGHTright- $c$ is geometrically not possible. Consider three oriented points $o_{A}, o_{B}$ and $o_{C}$ with $o_{A}$ SAMEright $o_{B}$ and

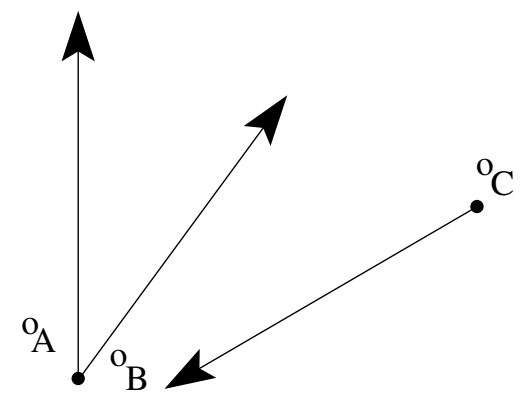

Figure 13: $\mathcal{O P} \mathcal{P} \mathcal{A}_{1}^{*}$ configuration

$o_{B}$ RIGHTright $+o_{C}$, as depicted in Fig. 13. In the picture, $o_{A}$ RIGHTright $+o_{C}$. For the relation $o_{A}$ RIGHTright- $o_{C}$ to hold, $o_{C}$ would need to be turned counter-clockwise. But turn would lead to first $o_{B}$ RIGHTrightA $o_{C}$ and then $o_{B}$ RIGHTright- $o_{C}$, even before $o_{A}$ RIGHTright- $o_{C}$ is reached.

The next result shows that we also can use quotients to transfer an important property of calculi.

Proposition 39 (refined from Moratz et al. 2009; Lücke 2012). Quotient homomorphisms of weak representations that are bijective in the second component preserve strength of composition.

12. This has already been reported (Moratz et al., 2009; Lücke, 2012). While the actual computation of the table was done with the same congruence relation as here, the quotient construction was wrong, resulting in a one-point algebra, as stated above.

13. See https://github.com/dwolter/SparQ/commit/89bebfc60a and https://github.com/dwolter/ SparQ/commit/dad260edd9. 


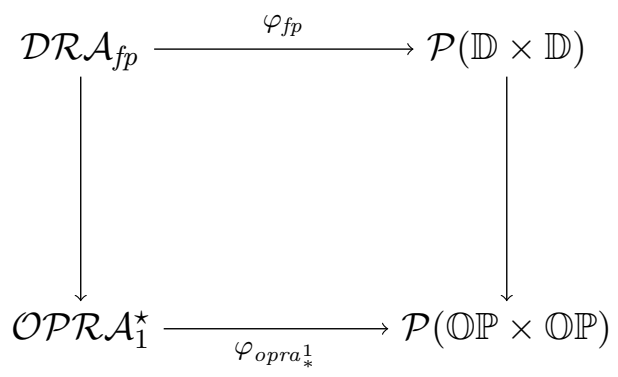

Figure 14: Quotient homomorphism of weak representations from $\mathcal{D} \mathcal{R} \mathcal{A}_{f p}$ to $\mathcal{O P} \mathcal{R} \mathcal{A}_{1}^{*}$

Proof. Let $(h, i): \varphi \rightarrow \psi$ with $\varphi: A \rightarrow \mathcal{P}(\mathcal{U} \times \mathcal{U})$ and $\psi: B \rightarrow \mathcal{P}(\mathcal{V} \times \mathcal{V})$ be a quotient homomorphism of weak representations such that $i$ is bijective. According to Prop. 23, the strength of the composition is equivalent to $\varphi$ (respectively $\psi$ ) being a proper homomorphism. We assume that $\varphi$ is a proper homomorphism and need to show that $\psi$ is proper as well. We also know that $h$ and $\mathcal{P}(i \times i)$ are proper. Let $R_{2}, S_{2}$ be two abstract relations in $B$. By surjectivity of $h$, there are abstract relations $R_{1}, S_{1} \in A$ with $h\left(R_{1}\right)=R_{2}$ and $h\left(S_{1}\right)=S_{2}$. Now $\psi\left(R_{2} \diamond S_{2}\right)=\psi\left(h\left(R_{1}\right) \diamond h\left(S_{1}\right)\right)=\psi\left(h\left(R_{1} \diamond S_{1}\right)\right)=\mathcal{P}(i \times i)\left(\varphi\left(R_{1} \diamond S_{1}\right)\right)=$ $\mathcal{P}(i \times i)\left(\varphi\left(R_{1}\right)\right) \diamond \mathcal{P}(i \times i)\left(\varphi\left(S_{1}\right)\right)=\psi\left(h\left(R_{1}\right)\right) \diamond \psi\left(h\left(S_{1}\right)\right)=\psi\left(R_{2}\right) \diamond \psi\left(S_{2}\right)$, hence $\psi$ is proper.

Corollary 40 (Moratz et al. 2009; Lücke 2012). Composition in $\mathcal{O P} \mathcal{R} \mathcal{A}_{1}^{*}$ is strong.

Proof. Composition in $\mathcal{D} \mathcal{R} \mathcal{A}_{f p}$ is known to be strong (Moratz, Lücke, \& Mossakowski, 2011). By Example 38 and Prop. 39, the strength of composition carries over to $\mathcal{O P} \mathcal{R} \mathcal{A}_{1}^{*}$.

Corollary 41. Composition in $\mathcal{C Y C}_{b}$ is strong.

Example 42. The quotient homomorphism of Example 31 has a one-sided inverse, namely the embedding (i.e. a proper injective homomorphism) of $\mathcal{C} \mathcal{Y} \mathcal{C}_{b}$ into $\mathcal{O P \mathcal { R }} \mathcal{A}_{1}^{*}$ At the level of non-associative algebras, the quotient is given by the table in Fig. 15. At the level of domains, it acts as follows: An orientation is mapped to the oriented point at $(0,0)$ with that orientation.

Altogether, we get the diagram of calculi (semi-strong representations) and homomorphisms in Fig. 16.

\section{Constraint Reasoning}

Let us now apply the relation-algebraic method to constraint reasoning. Given a nonassociative algebra $A$, a constraint network is a map $\nu: N \times N \rightarrow A$, where $N$ is a set of nodes (or variables) (Ligozat \& Renz, 2004). Individual constraints $\nu(X, Y)=R$ are written as $X \mathrm{R} Y$, where $X, Y$ are variables in $N$ and $\mathrm{R}$ is a relation in $A$. A constraint network $\nu: N \times N \rightarrow A$ is atomic or a scenario, if each $\nu(X, Y)$ is a base-relation. 


$$
\begin{aligned}
\mathrm{o} & \mapsto \text { SAMEback } \\
\mathrm{l} & \mapsto \text { SAMEleft } \\
\mathrm{r} & \mapsto \text { SAMEright } \\
\mathrm{e} & \mapsto \text { SAMEfront }
\end{aligned}
$$

Figure 15: Mapping from $\mathcal{C Y \mathcal { C }}$ to $\mathcal{O P} \mathcal{R} \mathcal{A}_{1}^{*}$ relations

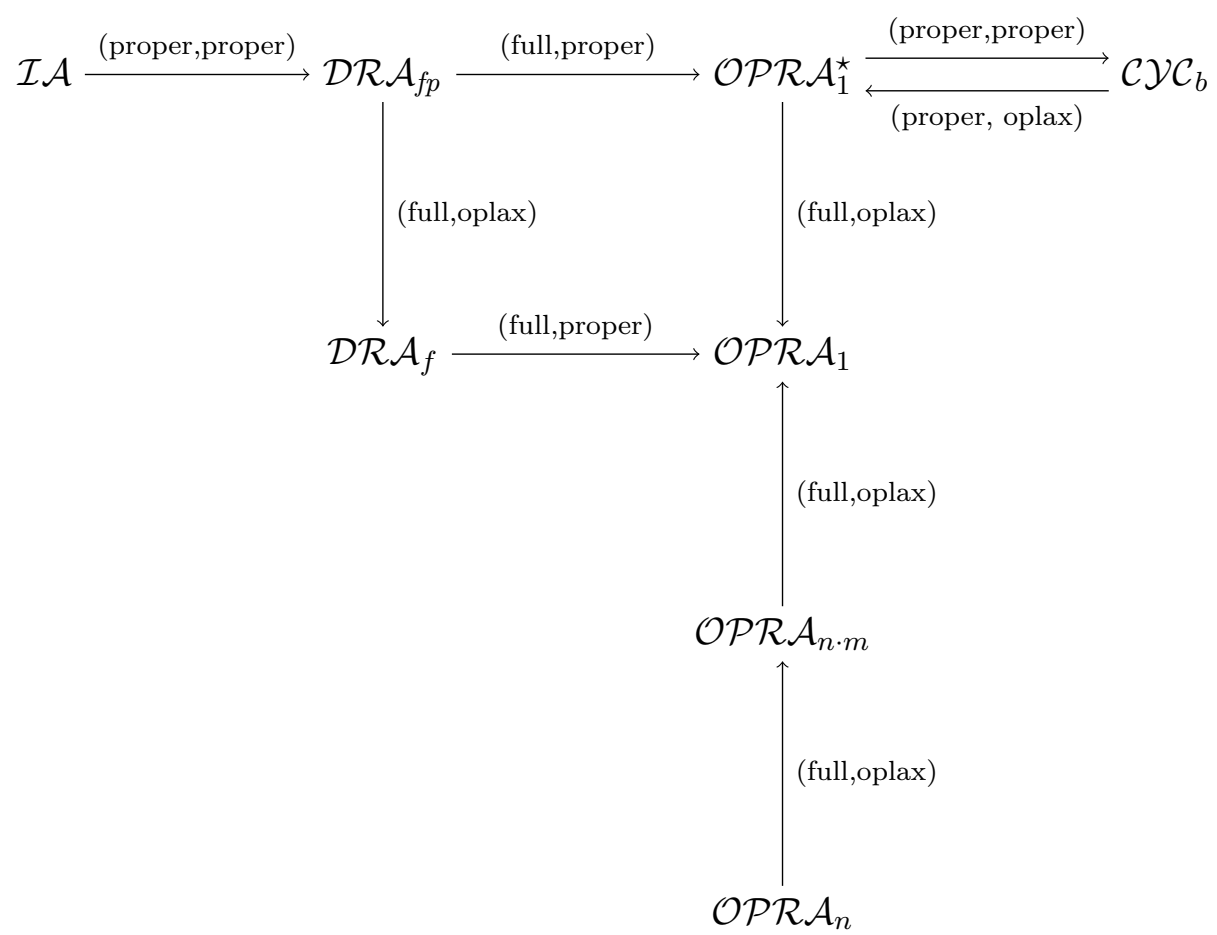

Figure 16: Homomorphisms among various calculi.

A constraint network $\nu$ is consistent if there is an assignment of all variables of $\nu$ with elements in the domain such that all constraints are satisfied (a solution). This problem is a Constraint Satisfaction Problem (CSP) (Mackworth, 1977). We rely on relation algebraic methods to check consistency, namely the above mentioned path consistency algorithm. For non-associative algebras, the abstract composition of relations need not coincide with the (associative) set-theoretic composition. Hence, in this case, the standard path-consistency algorithm does not necessarily lead to path consistent networks, but only to algebraic closure (Renz \& Ligozat, 2005):

Definition 43 (Algebraic Closure). A constraint network over binary relations is called algebraically closed if for all variables $X_{1}, X_{2}, X_{3}$ and all relations $\mathrm{R}_{1}, \mathrm{R}_{2}, \mathrm{R}_{3}$ the constraint 
relations

$$
X_{1} \mathrm{R}_{1} X_{2}, \quad X_{2} \mathrm{R}_{2} X_{3}, \quad X_{1} \mathrm{R}_{3} X_{3}
$$

imply

$$
\mathrm{R}_{3} \leq \mathrm{R}_{1} \diamond \mathrm{R}_{2}
$$

Algebraic closure can be enforced by successively applying

$$
\mathrm{R}_{3}:=\mathrm{R}_{3} \wedge\left(\mathrm{R}_{1} \diamond \mathrm{R}_{2}\right)
$$

for $X_{1} \mathrm{R}_{1} X_{2}, X_{2} \mathrm{R}_{2} X_{3}, X_{1} \mathrm{R}_{3} X_{3}$ until a fixed point is reached. Note that this procedure leaves the set of solutions of the constraint network invariant. This means that if the algebraic closure contains the empty relation, the original network is inconsistent. ${ }^{14}$

However, in general, algebraic closure is only a one-sided approximation of consistency: if algebraic closure detects an inconsistency, then we are sure that the constraint network is inconsistent; however, algebraic closure may fail to detect some inconsistencies: an algebraically closed network is not necessarily consistent. For some calculi, like Allen's interval algebra, algebraic closure is known to exactly decide consistency of scenarios, for others it does not (Renz \& Ligozat, 2005). It is also shown that this question is completely orthogonal to the question whether the composition is strong.

Constraint networks can be translated along homomorphisms of non-associative algebras as follows: Given $h: A \longrightarrow B$ and $\nu: N \times N \longrightarrow A$, let $h(\nu)$ be the composition $h \circ \nu$. It turns out that oplax homomorphisms preserve algebraic closure.

Proposition 44 (refined from Moratz et al. 2009; Lücke 2012). Given non-associative algebras $A$ and $B$, an oplax homomorphism $h: A \longrightarrow B$ preserves algebraic closure. An injective lax homomorphism reflects algebraic closure.

Proof. Since an oplax homomorphism is a homomorphism between Boolean algebras, it preserves the order. So for any three relations for $X_{1} \mathrm{R}_{1} X_{2}, X_{2} \mathrm{R}_{2} X_{3}, X_{1} \mathrm{R}_{3} X_{3}$ in the algebraically closed constraint network over $A$, with

$$
R_{3} \leq R_{1} \diamond R_{2}
$$

preservation of the order implies:

$$
h\left(R_{3}\right) \leq h\left(R_{1} \diamond R_{2}\right)
$$

Applying the oplaxness property yields:

$$
h\left(R_{3}\right) \leq h\left(R_{1}\right) \diamond h\left(R_{2}\right) .
$$

and hence the image of the constraint network under $h$ is also algebraically closed. If $h$ is injective and lax, it reflects equations and inequalities, and the converse implication follows in a similar way.

14. For scenarios, it suffices to check whether the scenario is algebraically closed, because any proper refinement must contain the empty relation. 
Given a scenario $\nu: N \times N \rightarrow A$, following Renz and Ligozat (2005), we can reorganize it as a function $\rho: A \rightarrow \mathcal{P}(N \times N)$ by defining $\rho(b)=\{(X, Y) \in N \times N \mid \nu(X, Y)=b\}$ for base-relations $b$ and extending this to all relations using joins as usual. Note that $\rho$ is a weak representation iff the scenario is algebraically closed and normalised. Here, a constraint network is normalised if $\nu(X, X)=\Delta$ and $\nu(Y, X)=\nu(X, Y)^{\smile}$.

For atomic homomorphisms (i.e. those mapping atoms to atoms), the translation of constraint networks can be lifted to scenarios represented as $\rho: A \rightarrow \mathcal{P}(N \times N)$ using the above correspondence, we then obtain $h(\rho): B \rightarrow \mathcal{P}(N \times N)$.

Definition 45. Given a scenario $\rho: A \rightarrow \mathcal{P}(N \times N)$, a solution for $\rho$ in a weak representation $\varphi: A \rightarrow \mathcal{P}(\mathcal{U} \times \mathcal{U})$ is a function $j: N \rightarrow \mathcal{U}$ such that for all $R \in A, \mathcal{P}(j \times j)(\rho(R)) \subseteq \varphi(R)$, or $\mathcal{P}(j \times j) \circ \rho \subseteq \varphi$ for short:

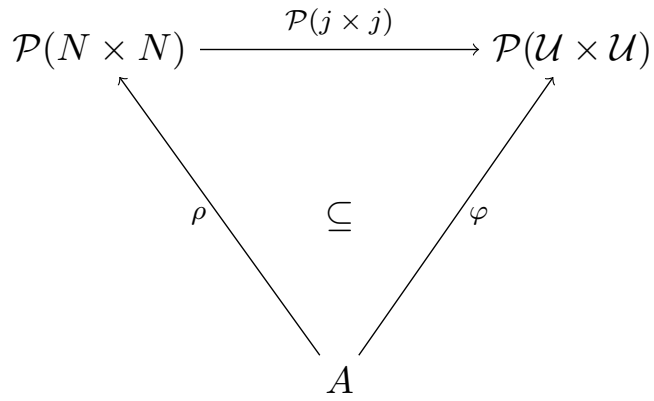

Proposition 46 (refined from Moratz et al. 2009; Lücke 2012). (_,oplax) homomorphisms of weak representations preserve solutions for scenarios.

Proof. Let weak representations $\varphi: A \rightarrow \mathcal{P}(\mathcal{U} \times \mathcal{U})$ and $\psi: B \rightarrow \mathcal{P}(\mathcal{V} \times \mathcal{V})$ and an (_,oplax) homomorphism of weak representations $(h, i): \varphi \rightarrow \psi$ be given.

A given solution $j: N \rightarrow \mathcal{U}$ for $\rho$ in $\varphi$ is defined by $\mathcal{P}(j \times j) \circ \rho \subseteq \varphi$. From this and the oplax commutation property $\mathcal{P}(i \times i) \circ \varphi \subseteq \psi \circ h$ we infer $\mathcal{P}(i \circ j \times i \circ j) \circ \rho \subseteq \psi \circ h$, which implies that $i \circ j$ is a solution for $h(\rho)$.

An important question for a calculus (= weak representation) is whether algebraic closure decides consistency of scenarios (Renz \& Ligozat, 2005). (Note that in general, any consistent scenario is algebraically closed, but not vice versa.) We will now prove that this property is preserved under certain homomorphisms.

Proposition 47 (refined from Moratz et al. 2009; Lücke 2012). Atomic (lax,oplax) homomorphisms $(h, i)$ of weak representations with injective $h$ preserve the following property to the image of $h$ :

\section{Algebraic closure decides scenario-consistency.}

Proof. Let weak representations $\varphi: A \rightarrow \mathcal{P}(\mathcal{U} \times \mathcal{U})$ and $\psi: B \rightarrow \mathcal{P}(\mathcal{V} \times \mathcal{V})$ and an atomic oplax homomorphism of weak representations $(h, i): \varphi \rightarrow \psi$ be given. Further assume that for $\varphi$, algebraic closure decides consistency of scenarios. 
Any scenario in the image of $h$ can be written as $h(\rho): B \rightarrow \mathcal{P}(N \times N)$. If $h(\rho)$ is algebraically closed, then by Prop. 44 , so is $\rho$. Hence, by the assumption, $\rho$ is consistent, i.e. has a solution. By Prop. $46, h(\rho)$ is consistent as well.

The general scenario consistency problem for the $\mathcal{D} \mathcal{R} \mathcal{A}_{f p}$ calculus is NP-hard and even $\exists \mathbb{R}$-complete (Wolter \& Lee, 2010; Lee, 2014). However, for specific scenarios, we can do better: We can apply Prop. 47 to the homomorphism from interval algebra to $\mathcal{D} \mathcal{R} \mathcal{A}_{f p}$ (see Example 27) and obtain:

Proposition 48 (Moratz et al. 2009; Lücke 2012). Algebraic closure decides consistency of $\mathcal{D R} \mathcal{A}_{f p}$ scenarios that involve the interval algebra relations only.

Hence, consistency of such scenarios can be decided in polynomial time (in spite of the NP-hardness of the general scenario consistency problem). A similar remark holds for the $\mathcal{C Y C}_{b}$ relations embedded into $\mathcal{O P \mathcal { R }} \mathcal{A}_{1}^{*}$.

For calculi such as RCC8, the interval algebra etc., (maximal) tractable subsets have been determined, i.e. sets of relations for which algebraic closure decides consistency also of non-atomic constraint networks involving these relations. It follows then that algebraic closure in $\mathcal{D} \mathcal{R} \mathcal{A}_{f p}$ decides consistency of any constraint network involving (the homomorphic image of) a maximal tractable subset of the interval algebra only.

\section{Conclusion}

Our study investigated calculi which on the application side represent the same modality on different levels of granularity. This modality in our case is relative direction. We demonstrated how to model relative directions on different levels of granularity with $\mathcal{D} \mathcal{R} \mathcal{A}_{f p}$, $\mathcal{O P R} \mathcal{A}_{1}^{*}$, and $\mathcal{C} \mathcal{Y} \mathcal{C}_{b}$. It turned out that in our case study of relative direction between oriented objects the formal relation between the calculi could be expressed as quotient homomorphisms.

This result is a step in the application of universal algebraic methods to qualitative constraint reasoning. Since there has been an explosion of qualitative constraint calculi in the recent years it becomes important to study the relations between those calculi and to make automatic mappings between the calculi. This is where we contribute with the presented work. We also have contributed a new notion of quotient (based on so-called oplax homomorphisms) between relation algebras that captures existing natural quotients between spatial calculi. We have published Haskell tools used for finding and checking homomorphisms between calculi in a public repository. ${ }^{15}$

As concrete results of our study we demonstrated how to answer questions whether composition is strong or algebraic closure decides consistency for calculi in which this has not been examined yet. With purely algebraic methods, we can lift the properties of strength of composition and of algebraic closure deciding consistency along homomorphisms of qualitative calculi. The latter is particularly important, because algebraic closure is a polynomialtime method, whereas qualitative constraint problems in some cases turn out to be NP-hard, even for scenarios of base-relations.

15. See https://github.com/spatial-reasoning/homer 
We derived a chain of calculi and homomorphisms between $\mathcal{D} \mathcal{R} \mathcal{A}_{f p}, \mathcal{O P} \mathcal{R} \mathcal{A}_{1}^{*}, \mathcal{C} \mathcal{Y} \mathcal{C}_{b}$. Thereby we combined the dipole and opra calculi with the cycord approach. Based on this new approach we could automatically derive a composition table for $\mathcal{O P} \mathcal{R} \mathcal{A}_{1}^{*}$ based on the formally verified composition table of $\mathcal{D} \mathcal{R} \mathcal{A}_{f p}$. We compared this table with the composition table for $\mathcal{O P} \mathcal{R} \mathcal{A}_{1}^{*}$ described in previous work by other authors (Dylla, 2008). It turned out that this old composition table as it was shipped with the qualitative reasoner SparQ contained errors in 197 entries. This emphasizes our point how important it is to develop a sound mathematical theory as a basis for the computation of composition tables and to stay as close as possible with the implementation to the theory.

\section{Acknowledgements}

The authors would like to thank Dominik Lücke, André van Delden, Torsten Hahmann, Jay Lee, Thomas Schneider and Diedrich Wolter for fruitful discussions and Thomas Schneider for valuable comments on a draft. Also the anonymous referees provided valuable hints. Our work was supported by the DFG Transregional Collaborative Research Center SFB/TR 8 "Spatial Cognition", projects I4-[SPIN] and R4-[LogoSpace] (TM), and the National Science Foundation under Grant No. CDI-1028895 (RM).

\section{References}

Allen, J. F. (1983). Maintaining knowledge about temporal intervals. Communications of the ACM, pages 832-843, 1983.

Bodirsky, M. (2008). Constraint satisfaction problems with infinite templates. In Creignou, N., Kolaitis, P. G., \& Vollmer, H., editors, Complexity of Constraints - An Overview of Current Research Themes [Result of a Dagstuhl Seminar]., volume 5250 of Lecture Notes in Computer Science, pages 196-228. Springer, 2008. ISBN 978-3-540-92799-0. doi: 10. 1007/978-3-540-92800-3_8. URL http://dx.doi.org/10.1007/978-3-540-92800-3_8.

Burmeister, P. (1986). A model theoretic approach to partial algebras. Akademie Verlag, Berlin, 1986.

Burmeister, P. (2002). Lecture notes on universal algebra many-sorted partial algebras preliminary version. see http://www.mathematik.tu-darmstadt.de/Math-Net/ Lehrveranstaltungen/Lehrmaterial/SS2002/AllgemeineAlgebra/download/ LNPartAlg.pdf, 2002.

Clementini, E., Felice, P. D., \& Hernandez, D. (1997). Qualitative Represenation of Positional Information. Artificial Intelligence, 95:317-356, 1997.

Dubba, K. S. R., Bhatt, M., Dylla, F., Cohn, A. G., \& Hogg, D. C. (2015). Learning relational event models from video. J. Artif. Intell. Res. (JAIR), 52, 2015. accepted for publication.

Düntsch, I. (2005). Relation algebras and their application in temporal and spatial reasoning. Artif. Intell. Rev., 23(4):315-357, 2005. 
Dylla, F. (2008). An Agent Control Perspective on Qualitative Spatial Reasoning - Towards More Intuitive Spatial Agent Development. PhD thesis, University of Bremen, 2008. Published by Akademische Verlagsgesellschaft Aka GmbH.

Dylla, F., Mossakowski, T., Schneider, T., \& Wolter, D. (2013). Algebraic properties of qualitative spatio-temporal calculi. In Tenbrink, T., Stell, J. G., Galton, A., \& Wood, Z., editors, COSIT, volume 8116 of Lecture Notes in Computer Science, pages 516-536. Springer, 2013. ISBN 978-3-319-01789-1. doi: 10.1007/978-3-319-01790-7. URL http: //dx.doi.org/10.1007/978-3-319-01790-7.

Egenhofer, M. \& Franzosa, R. (1991). Point-Set Topological Spatial Relations. International Journal of Geographical Information Systems, 5(2):161-174, 1991.

Frank, A. (1991). Qualitative Spatial Reasoning with Cardinal Directions. In Kaindl, H., editor, Proc. of 7th Österreichische Artificial-Intelligence-Tagung, pages 157-167. Springer, 1991.

Freksa, C. (1992). Using orientation information for qualitative spatial reasoning. In Frank, A. U., Campari, I., \& Formentini, U., editors, Theories and methods of spatio-temporal reasoning in geographic space, volume 639 of Lecture Notes in Comput. Sci., pages 162178. Springer, 1992.

Gantner, Z., Westphal, M., \& Wölfl, S. (2008). GQR - A Fast Reasoner for Binary Qualitative Constraint Calculi. In Proc. of the AAAI-08 Workshop on Spatial and Temporal Reasoning, 2008.

Grätzer, G. (1979). Universal Algebra. Springer-Verlag, New York, NY, second edition, 1979.

Hobbs, J. R. (1985). Granularity. In In Proceedings of the Ninth International Joint Conference on Artificial Intelligence, 1985.

Huang, J. (2012). Compactness and its implications for qualitative spatial and temporal reasoning. In Brewka, G., Eiter, T., \& McIlraith, S. A., editors, Principles of Knowledge Representation and Reasoning: Proceedings of the Thirteenth International Conference, KR 2012, Rome, Italy, June 10-14, 2012. AAAI Press, 2012. ISBN 978-1-57735-560-1. URL http://www . aaai.org/ocs/index.php/KR/KR12/paper/view/4494.

Isli, A. \& Cohn, A. G. (2000). A new approach to cyclic ordering of 2 D orientations using ternary relation algebras. Artificial Intelligence, 122(1-2):137-187, 2000.

Ladkin, P. \& Maddux, R. (1994). On Binary Constraint Problems. J. ACM, 41(3):435-469, 1994.

Lee, J. H. (2014). The complexity of reasoning with relative directions. In Schaub, T., Friedrich, G., \& O'Sullivan, B., editors, ECAI 2014 - 21st European Conference on 
Artificial Intelligence, 18-22 August 2014, Prague, Czech Republic - Including Prestigious Applications of Intelligent Systems (PAIS 2014), volume 263 of Frontiers in Artificial Intelligence and Applications, pages 507-512. IOS Press, 2014. ISBN 978-161499-418-3. doi: 10.3233/978-1-61499-419-0-507. URL http://dx.doi.org/10.3233/ 978-1-61499-419-0-507.

Li, J. J., Kowalski, T., Renz, J., \& Li, S. (2008). Combining binary constraint networks in qualitative reasoning. In Ghallab, M., Spyropoulos, C. D., Fakotakis, N., \& Avouris, N. M., editors, ECAI 2008 - 18th European Conference on Artificial Intelligence, Patras, Greece, July 21-25, 2008, Proceedings, volume 178 of Frontiers in Artificial Intelligence and Applications, pages 515-519. IOS Press, 2008. ISBN 978-1-58603-891-5. doi: 10.3233/ 978-1-58603-891-5-515. URL http://dx.doi.org/10.3233/978-1-58603-891-5-515.

Ligozat, G. (1993). Qualitative triangulation for spatial reasoning. In Frank, A. U. \& Campari, I., editors, Proc. International Conference on Spatial Information Theory., volume 716 of Lecture Notes in Comput. Sci., pages 54-68. Springer, 1993.

Ligozat, G. (1998). Reasoning about Cardinal Directions. J. Vis. Lang. Comput., 9(1): 23-44, 1998.

Ligozat, G. (2005). Categorical Methods in Qualitative Reasoning: The Case for Weak Representations. In Cohn, A. G. \& Mark, D. M., editors, Proc. of COSIT, volume 3693 of Lecture Notes in Comput. Sci., pages 265-282. Springer, 2005.

Ligozat, G. \& Renz, J. (2004). What Is a Qualitative Calculus? A General Framework. In Zhang, C., Guesgen, H. W., \& Yeap, W.-K., editors, Proc. of PRICAI-04, pages 53-64, 2004 .

Ligozat, G. (2011). Qualitative Spatial and Temporal Reasoning. Wiley, 2011. ISBN $9781848212527,9781118601457$.

Lücke, D. (2012). Qualitative Spatial Reasoning about Relative Orientation: A Question of Consistency. PhD thesis, University of Bremen, 2012. http://elib.suub.uni-bremen. de/edocs/00102632-1.pdf.

Mackworth, A. K. (1977). Consistency in Networks of Relations. Artif. Intell., 8:99-118, 1977.

Maddux, R. (2006). Relation Algebras. Stud. Logic Found. Math. Elsevier Science, 2006.

Montanari, U. (1974). Networks of constraints: Fundamental properties and applications to picture processing. Inf. Sci., 7:95-132, 1974.

Moratz, R. (2006). Representing Relative Direction as a Binary Relation of Oriented Points. In Brewka, G., Coradeschi, S., Perini, A., \& Traverso, P., editors, Proc. of ECAI-06, volume 141 of Frontiers in Artificial Intelligence and Applications, pages 407-411. IOS Press, 2006.

Moratz, R., Renz, J., \& Wolter, D. (2000). Qualitative Spatial Reasoning about Line Segments. In Proc. of ECAI 2000, pages 234-238, 2000. 
Moratz, R., Lücke, D., \& Mossakowski, T. (2009). Oriented straight line segment algebra: Qualitative spatial reasoning about oriented objects. CoRR, abs/0912.5533, 2009. URL http://arxiv.org/abs/0912.5533.

Moratz, R., Lücke, D., \& Mossakowski, T. (2011). A condensed semantics for qualitative spatial reasoning about oriented straight line segments. Artif. Intell., 175(16-17):20992127, 2011.

Mossakowski, T., Schröder, L., \& Wölfl, S. (2006). A categorical perspective on qualitative constraint calculi. In Wölfl, S. \& Mossakowski, T., editors, Qualitative Constraint Calculi - Application and Integration. Workshop at KI 2006, pages 28-39, 2006.

Mossakowski, T. \& Moratz, R. (2012). Qualitative reasoning about relative direction of oriented points. Artificial Intelligence, 180-181(0):34 - 45, 2012.

Nebel, B. \& Wölfl, S., editors (2009). AAAI Spring Symposium on Benchmarking of Qualitative Spatial and Temporal Reasoning Systems. AAAI Technical Report SS-09-02, 2009.

Randell, D. A. \& Cohn, A. G. (1989). Modelling topological and metrical properties of physical processes. In Brachman, R. J., Levesque, H. J., \& Reiter, R., editors, Proc. of KR-89, pages 357-368. Morgan Kaufmann, 1989.

Randell, D. A., Cui, Z., \& Cohn, A. G. (1992). A spatial logic based on regions and connection. In Nebel, B., Rich, C., \& Swartout, W., editors, Proc. of KR-92, pages 165-176. Morgan Kaufmann, 1992.

Renz, J. \& Ligozat, G. (2005). Weak Composition for Qualitative Spatial and Temporal Reasoning. In van Beek, P., editor, Proc. of CP-05, volume 3709 of Lecture Notes in Comput. Sci., pages 534-548. Springer, 2005.

Renz, J. \& Mitra, D. (2004). Qualitative Direction Calculi with Arbitrary Granularity. In Zhang, C., Guesgen, H. W., \& Yeap, W.-K., editors, Proc. of PRICAI-04, volume 3157 of Lecture Notes in Comput. Sci., pages 65-74. Springer, September 2004.

Renz, J. \& Nebel, B. (1999). On the Complexity of Qualitative Spatial Reasoning: A Maximal Tractable Fragment of the Region Connection Calculus. Artificial Intelligence, 108(1-2):69-123, 1999.

Renz, J. \& Nebel, B. (2007). Qualitative Spatial Reasoning Using Constraint Calculi. In Aiello, M., Pratt-Hartmann, I., \& van Benthem, J., editors, Handbook of Spatial Logics, pages 161-215. Springer, 2007.

Scivos, A. \& Nebel, B. (2004). The finest of its class: The natural point-based ternary calculus for qualitative spatial reasoning. In Freksa, C., Knauff, M., Brückner, B. K., Nebel, B., \& T.Barkowski, editors, Spatial Cognition, volume 3343 of Lecture Notes in Comput. Sci., pages 283-303. Springer, 2004.

van Beek, P. \& Manchak, D. W. (1996). The design and experimental analysis of algorithms for temporal reasoning. J. Artif. Intell. Res., 4:1-18, 1996. 
Wallgrün, J. O., Frommberger, L., Wolter, D., Dylla, F., \& Freksa, C. (2006). Qualitative Spatial Representation and Reasoning in the SparQ-Toolbox. In Barkowsky, T., Knauff, M., Ligozat, G., \& Montello, D. R., editors, Spatial Cognition, volume 4387 of Lecture Notes in Comput. Sci., pages 39-58. Springer, 2006.

Wallgrün, J. O., Frommberger, L., Dylla, F., \& Wolter, D. (2009). SparQ User Manual V0.7. User manual, University of Bremen, January 2009.

Westphal, M. \& Wölfl, S. (2009). Qualitative CSP, finite CSP, and SAT: Comparing methods for qualitative constraint-based reasoning. In Boutilier, C., editor, IJCAI, pages 628-633, 2009 .

Wolter, D. \& Lee, J. H. (2010). Qualitative reasoning with directional relations. Artificial Intelligence, 174(18):1498-1507, 2010. doi: 10.1016/j.artint.2010.09.004.

Worboys, M. F. \& Clementini, E. (2001). Integration of Imperfect Spatial Information. Journal of Visual Languages and Computing, 12:61-80, 2001. 